\title{
The Moduli Space of Real Abelian Varieties with Level Structure
}

\author{
MARK GORESKY ${ }^{1 \star}$ and YUNG SHENG TAI ${ }^{2}$ \\ ${ }^{1}$ School of Mathematics, Institute for Advanced Study, Princeton N.J., U.S.A. \\ email: goresky@math.ias.ed. \\ ${ }^{2}$ Department of Mathematics, Haverford College, Haverford PA, U.S.A.
}

(Received: 18 October 2001; accepted in final form 3 June 2002)

\begin{abstract}
The moduli space of principally polarized Abelian varieties with real structure and with level $N=4 m$ structure (with $m \geqslant 1$ ) is shown to coincide with the set of real points of a quasi-projective algebraic variety defined over $\mathrm{Q}$, and to consist of finitely many copies of the quotient of the space $\mathrm{GL}(n, \mathrm{R}) / \mathrm{O}(N)$ (of positive definite symmetric matrices) by the principal congruence subgroup of level $N$ in $\operatorname{GL}(n, Z)$.
\end{abstract}

Mathematics Subject Classification (2000). Primary: 14K10, 14P05, 53C35; Secondary: 11G10, 14L35, 20G20.

Key words. Abelian variety, real algebraic, symmetric space, arithmetic group, antiholomorphic involution, moduli space, Comessatti lemma, Baily-Borel compactification.

\section{Introduction}

1.1. Let $\mathfrak{h}_{n}=\operatorname{Sp}(2 n, \mathbb{R}) / \mathrm{U}(n)$ be the Siegel upperhalf space of rank $n$. The quotient space $\operatorname{Sp}(2 n, Z) \backslash \mathfrak{h}_{n}$ has three remarkable properties: (a) it is the moduli space of principally polarized Abelian varieties, (b) it has the structure of a quasi-projective complex algebraic variety which is defined over the rational numbers $Q$, and (c) it has a natural compactification (the Baily-Borel Satake compactification) which is defined over the rational numbers.

Now let $C_{n}=\mathrm{GL}(n, \mathbb{R}) / \mathrm{O}(n)$ be the symmetric cone of positive definite symmetric matrices and let $Z=\mathrm{GL}(n, Z) \backslash C_{n}$. One might ask whether similar statements hold for $Z$. Is $Z$ in some sense a moduli space for principally polarized real Abelian varieties? Does it admit the structure of a real algebraic variety, possibly defined over Q? If so, does it admit a compactification which is also defined over the rational numbers? The answer to all these questions is 'no'. In fact, Silhol [Si] constructs the moduli space of real principally polarized Abelian varieties and he shows that it is a (topological) ramified covering of $Z$. Moreover Silhol constructs a compactification of this moduli space, analogous to the Baily-Borel compactification. However, neither the moduli space nor this compactification has an algebraic structure.

\footnotetext{
${ }^{\star}$ Research partially supported by NSF grants \# DMS 9900324 and DMS 0139986.
} 
In this paper we show that all three statements (a), (b), and (c) above may be recovered if we consider real Abelian varieties with an appropriate level structure. To be precise, let $N=4 m$ for some $m \geqslant 1$, let $\Gamma_{\ell}(N)$ be the principal congruence subgroup of GL $(n, Z)$ of level $N$, and let $Z(N)=\Gamma_{\ell}(N) \backslash C_{n}$. Then (a) the moduli space of real principally polarized Abelian varieties with level $N$ structure consists of finitely many copies of $Z(N)$. These various copies are indexed by a certain (nonAbelian cohomology) set $H^{1}(\mathrm{C} / \mathbb{R}, \Gamma(N))$. Morever, (b) this moduli space may be naturally identified with the real points $X_{\mathrm{R}}$ of a quasi-projective algebraic variety $X$ which is defined over the rational numbers. See Theorems 4.2 and 6.6. Finally, (c) the variety $X_{\mathrm{C}}$ has a compactification which is defined over $\mathrm{Q}$.

1.2. In fact the variety $X_{\mathrm{C}}$ is just the moduli space $\Gamma(N) \backslash \mathfrak{G}_{n}$ of Abelian varieties with principal polarization and level $N=4 m$ structure. (Here, $\Gamma(N)$ denotes the principal congruence subgroup of $\mathrm{Sp}(2 n, \mathrm{Z})$ of level $N$.) The first observation is that there is an involution $\tau$ on $\operatorname{Sp}(2 n, \mathbb{R})$ whose fixed point set is $\operatorname{GL}(n, \mathbb{R})$, which passes to an antiholomorphic involution on $X_{\mathrm{C}}$. This implies that the set of real points of $X_{\mathrm{C}}$ consists of finitely many arithmetic quotients of the symmetric cone $C_{n}$. What is not so obvious, however, is that these quotients are copies of a single arithmetic quotient $\Gamma_{\ell}(N) \backslash C_{n}$ or that this set of real points may be realized as the moduli space of real principally polarized Abelian varieites with level $N$ structure. Indeed, these statements are likely false for $N \not \equiv 0(\bmod 4)$. The key technical tool (which is needed for both these facts) is the lemma of Comessatti and Silhol ([C, Si, Si2]) describing certain conditions on the period matrix of a principally polarized Abelian variety $A$ which are necessary in order for $A$ to admit a real structure.

1.3. Let $V=V_{\mathrm{C}}$ denote the Baily-Borel Satake compactification of $X_{\mathrm{C}}$. It is an algebraic variety defined over Q. Complex conjugation $\tau: V_{\mathrm{C}} \rightarrow V_{\mathrm{C}}$ is an antiholomorphic involution whose fixed point set is the set of real points $V_{\mathrm{R}}$. This set is compact and it contains $X_{\mathbb{R}}$ as an open set. One might ask whether $V_{\mathbb{R}}$ is a compactification of $X_{\mathrm{R}}$, that is, whether $V_{\mathbb{R}}=\bar{X}_{\mathbb{R}}$, where $\bar{X}_{\mathrm{R}}$ denotes the closure of $X_{\mathrm{R}}$ in $V_{\mathrm{C}}$. We have been able to prove this (Section 10) in the special case $N=2^{k}$ (that is, for the principal congruence subgroup $\Gamma\left(2^{k}\right)$ of level $2^{k}$ ) for $k \geqslant 2$, and we suspect it is otherwise false unless $n=1$; (cf. Section 5). However, we have been able to show (for general $\Gamma(4 m)$ ) that the complement $V_{\mathbb{R}}-\bar{X}_{\mathbb{R}}$ has a high codimension. The variety $V_{\mathrm{C}}$ has a stratification

$$
V_{\mathrm{C}}=V_{\mathrm{C}}^{0} \cup V_{\mathrm{C}}^{1} \cup \cdots \cup V_{\mathrm{C}}^{n}
$$

such that each $V_{\mathrm{C}}^{r}$ is a union of arithmetic quotients $\Gamma \backslash \mathfrak{h}_{n-r}$ of finitely many copies of the Siegel space of rank $n-r$. The largest stratum $V_{\mathrm{C}}^{0}$ is just $X_{\mathrm{C}}$. Denote by $V_{\mathrm{R}}^{r}$ the $\tau$-fixed points in $V_{\mathrm{C}}^{r}$. In Proposition 9.2 we show that

$$
V_{\mathbb{R}}^{0} \cup V_{\mathbb{R}}^{1} \subset \bar{X}_{\mathbb{R}} \subset V_{\mathbb{R}}
$$

Although the moduli space $X_{\mathrm{R}}$ consists of finitely many disjoint copies of the locally symmetric space $Z(N)$, the compactification $\bar{X}_{\mathbb{R}}$ is not a disjoint union: some of these copies of $Z(N)$ may become glued together along the boundary. 
1.4. The questions in the first paragraph, and others like them, were posed by G. Shimura, J. Millson and M. Kuga [J] in the early 1970's, in the context of attempts to associate automorphic forms on groups of Hermitian type to automorphic forms on groups of non-Hermitian type. One of many missing ingredients in the theory of automorphic forms for groups of non-Hermitian type arises from the fact that the associated locally symmetric space does not appear to have an algebraic structure, or to be associated with elliptic curves or Abelian varieties. On the other hand, suppose $X_{\mathrm{C}}$ is a moduli space for Abelian varieties with certain polarization, endomorphism, and level structures, and suppose that $X$ has a model defined over $\mathbb{R}$. In [Sh1], Shimura showed that the set of real points $X_{\mathbb{R}}$ does not necessarily give a moduli space for real Abelian varieties (with the corresponding PEL structures).

These questions were partially investigated by A. Adler [A], H. Jaffee [J], S. Kudla [K], M. Kuga, J. Millson, K.-Y. Shih [Shh], and G. Shimura [Sh1, Sh3, Sh4]. Kudla, for example, enumerated the possible real forms and determined the number of connected components of the set of real points of certain compact arithmetic quotients $\Gamma \backslash \mathfrak{G}^{n}$ of a product of upper half planes. Related results concerning products $\mathfrak{h}_{r}^{n}$ of Siegel upper half-spaces appear in [Sh3]. Since 1975 however, this line of investigation appears to have been abandoned. Indeed, it is unlikely that this work could have progressed much further without (some analog of) the lemma of Comessatti and Silhol. We believe Theorems 4.2 and 6.6, and the related results in [GT], are but two examples of a much more general phenomenon involving arithmetic quotients of symmetric cones associated to (formally real) Jordan algebras.

\section{Non-Abelian Cohomology}

2.1. Let $\mathbf{G}$ be a reductive algebraic group defined over $Q$, let $K$ be a maximal compact subgroup of $G=\mathbf{G}(\mathbb{R})$, and let $\Gamma \subset \mathbf{G}(Q)$ be an arithmetic subgroup. Set $D=G / K$ and $X=\Gamma \backslash G / K$ with projection $\pi: D \rightarrow X$. Suppose $\tau: G \rightarrow G$ is an involution which preserves $K$ and $\Gamma$. Then $\tau$ passes to an involution on $X$ and we denote by $X^{\tau}$ the fixed point set. Let $\langle\tau\rangle$ be the group $\{1, \tau\}$ and let $H^{1}(\langle\tau\rangle, \Gamma)$ be the first non-Abelian cohomology set. For any $\gamma \in \Gamma$ let $f_{\gamma}:\langle\tau\rangle \rightarrow \Gamma$ be the mapping $f_{\gamma}(1)=1$ and $f_{\gamma}(\tau)=\gamma$. Then $f_{\gamma}$ is a 1-cocycle iff $\gamma \tau(\gamma)=1$, in which case its cohomology class is denoted $\left[f_{\gamma}\right]$. A cocycle $f_{\gamma}$ is a coboundary iff there exists $h \in \Gamma$ so that $\gamma=\tau(h) h^{-1}$. To every such 1-cocycle $f_{\gamma}$ we associate the ' $\gamma$-twisted' involutions $\tau \gamma: D \rightarrow D$ by $x \mapsto \tau(\gamma x)$ and $\tau \gamma: \Gamma \rightarrow \Gamma$ by $\gamma^{\prime} \mapsto \tau\left(\gamma \gamma^{\prime} \gamma^{-1}\right)$. Let $D^{\tau \gamma}$ be the fixed point set in $D$ of the involution $\tau \gamma$ and let $\Gamma^{\tau \gamma}$ be the fixed group in $\Gamma$ of the involution $\tau \gamma$.

To describe $X^{\tau}$ it is necessary to understand the various $D^{\tau \gamma}:$ if $Z \in D$, then $\pi(Z) \in X^{\tau}$ iff there exists $\gamma \in \Gamma$ such that $Z \in D^{\tau \gamma}$. For completeness, we recall the proof of the following theorem ([Ro1, Ro2, RoS]) of Rohlfs.

THEOREM 2.2. Suppose $\Gamma$ is torsion free. Then the association

$$
f_{\gamma} \mapsto X(\tau \gamma)=\pi\left(D^{\tau \gamma}\right)=\Gamma^{\tau \gamma} \backslash D^{\tau \gamma}
$$


determines a one to one correspondence between the cohomology set $H^{1}(\langle\tau\rangle, \Gamma)$ and the connected components of the fixed point set $X^{\tau}$.

Proof 2.3. The twisted involution $\tau \gamma: D \rightarrow D$ acts by isometries so $([\mathrm{H}]$ Chapter $I$ Section 13.5) the fixed point set $D^{\tau \gamma}$ is nonempty. If $x, x^{\prime} \in D^{\tau \gamma}$ then the unique geodesic connecting them is also fixed by $\tau \gamma$, so $D^{\tau \gamma}$ is connected. Its image in $X$ is a connected component $X(\tau \gamma)$ of $X^{\tau}$ which depends only on the cohomology class of $f_{\gamma}$. It is easy to check that $f_{\gamma}$ and $f_{\gamma^{\prime}}$ are cohomologous iff $X(\tau \gamma) \cap X\left(\tau \gamma^{\prime}\right) \neq \phi$.

2.4. In general, the cohomology set $H^{1}(\langle\tau\rangle, \Gamma)$ may be difficult to compute, the connected component $X(\tau \gamma)$ may be difficult to describe, and distinct components may fail to be isomorphic. We will introduce additional hypotheses which will allow us to address these three issues. Let $\Gamma_{0} \subset \mathbf{G}(Q)$ be a $\tau$ stable arithmetic subgroup which contains $\Gamma$. Let $\theta: G \rightarrow G$ be the Cartan involution corresponding to $K$. Consider the following possible hypotheses (cf. Remark 2.7, Theorem 4.2 and Proposition 4.6).

(1) $G$ is Zariski connected, $\tau: \mathbf{G} \rightarrow \mathbf{G}$ is defined over $\mathbb{R}$, its fixed point set $G^{\tau}=\mathbf{G}^{\tau}(\mathbb{R})$ is Zariski connected, and $\theta$ preserves $G^{\tau}$,

(2) $H^{1}(\langle\tau\rangle, K)$ is trivial,

(3) $H^{1}(\langle\tau\rangle, \Gamma) \rightarrow H^{1}\left(\langle\tau\rangle, \Gamma_{0}\right)$ is trivial,

(4) $\Gamma_{0}$ contains $\Gamma$ as a normal subgroup,

(5) $\tau$ acts trivially on $\Gamma \backslash \Gamma_{0}$, and

(6) $\Gamma$ is torsion free.

We remark that if $\Gamma_{0}$ satisfies conditions (3) and (4) then there is an arithmetic group $\mathcal{G}$ with $\Gamma \subset \mathcal{G} \subset \Gamma_{0}$ which satisfies conditions (3), (4), and (5), namely

$$
\mathcal{G}=\mathcal{G}\left(\Gamma, \Gamma_{0}\right)=\left\{a \in \Gamma_{0} \mid \tau(a) a^{-1} \in \Gamma\right\}
$$

LEMMA 2.5. Suppose $\Gamma \subset \Gamma_{0} \subset \mathbf{G}(Q)$ are arithmetic subgroups. Then the following statements hold.

(a) Under hypothesis (1) above, $G^{\tau}$ is reductive, $\theta$ restricts to a Cartan involution of $G^{\tau}$, and $K^{\tau}$ is a maximal compact subgroup of $G^{\tau}$.

(b) Under hypothesis (2) above, $D^{\tau}=G^{\tau} / K^{\tau}$.

(c) Under hypothesis (3) above, for each cohomology class $\left[f_{\gamma}\right] \in H^{1}(\langle\tau\rangle, \Gamma)$ there exists $h \in \Gamma_{0}$ such that $\gamma=\tau(h) h^{-1}$, in which case,

$$
D^{\tau \gamma}=h D^{\tau} \text { and } \Gamma^{\tau \gamma}={ }^{h} \Gamma^{\tau}=h \Gamma^{\tau} h^{-1} .
$$

(d) Under hypotheses (3), (4), and (5) this association $f_{\gamma} \mapsto h$ determines a one to one correspondence

$$
H^{1}(\langle\tau\rangle, \Gamma) \cong \Gamma \backslash \Gamma_{0} / \Gamma_{0}^{\tau} .
$$

(e) Under hypotheses (3), (4), (5), and (6) the fixed point set $X^{\tau}$ is the disjoint union of 
isomorphic copies

$$
X^{\tau}=\coprod_{h \in \Gamma \backslash \Gamma_{0} / \Gamma_{0}^{\tau}}{ }^{h} \Gamma^{\tau} \backslash h D^{\tau}
$$

of the quotient $\Gamma^{\tau} \backslash D^{\tau}$.

Proof 2.6. Part (a) is proven in [Sa] Ch. I Theorem 4.2 and Corollary 4.5 (pages 15 and 17). Now consider part (b). Clearly, $G^{\tau} / K^{\tau} \subset D^{\tau}$ so it suffices to show that $G^{\tau}$ acts transitively on $D^{\tau}$. Let $x=g K \in D^{\tau}$. Then $\tau(g) K=g K$ so the element $k=g^{-1} \tau(g)$ lies in $K$. Moreover, $f_{k}$ is a cocycle, so by (2) there exists $u \in K$ with $k=u \tau(u)^{-1}=g^{-1} \tau(g)$. Then $g u=\tau(g u) \in G^{\tau}$ and $x=g u K$. Part (c) is straightforward. Part (d) follows from the long exact sequence

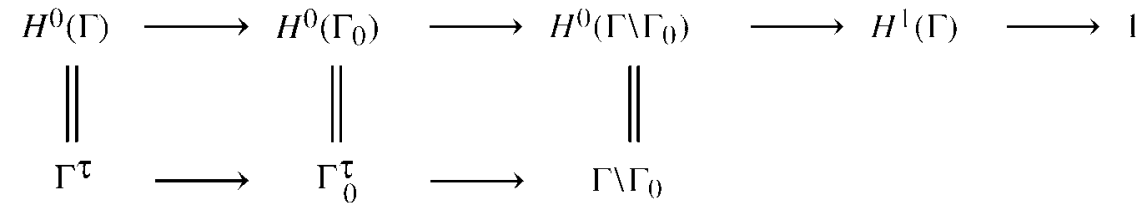

associated to the sequence $1 \rightarrow \Gamma \rightarrow \Gamma_{0} \rightarrow \Gamma \backslash \Gamma_{0} \rightarrow 1$. Part (e) follows from Rohlfs' theorem and parts (c) and (d) however it is also easy to verify directly. First check that $X^{\tau}=\pi\left(\bigcup_{h \in \Gamma_{0}} h D^{\tau}\right)$. If $h \in \Gamma_{0}$ then $\tau(h) h^{-1} \in \Gamma$ by (5), which implies that $\pi\left(h D^{\tau}\right) \subset X^{\tau}$. On the other hand, if $x \in D$ and $\pi(x) \in X^{\tau}$ then there exists $\gamma \in \Gamma$ such that $\tau(x)=\gamma x$. By (6), $f_{\gamma}$ is a cocycle, so by (3) there exists $h \in \Gamma_{0}$ such that $\gamma=\tau(h) h^{-1}$, hence $x \in h D^{\tau}$. To see that the union (2.5.1) is disjoint, suppose $\pi\left(h D^{\tau}\right) \cap \pi\left(h^{\prime} D^{\tau}\right) \neq \phi$. Then there exists $x, x^{\prime} \in D^{\tau}$ and $\gamma \in \Gamma$ such that $\gamma h x=h^{\prime} x^{\prime}$, hence $\tau(\gamma h) x=\tau\left(h^{\prime}\right) x^{\prime}$. This gives

$$
x=\tau\left(h^{-1} \gamma^{-1} h^{\prime}\right) x^{\prime}=\tau\left(h^{-1} \gamma^{-1} h^{\prime}\right)\left(h^{-1} \gamma^{-1} h^{\prime}\right)^{-1} x .
$$

For any $u \in \Gamma_{0},(5)$ implies that $\tau(u) u^{-1} \in \Gamma$, which is torsion free. Take $u=h^{-1} \gamma^{-1} h^{\prime}$. Then $\tau(u)=u$ so $u \in \Gamma_{0}^{\tau}$ and $h^{\prime}=\gamma h u \in \Gamma h \Gamma_{0}^{\tau}$.

Remark 2.7. If $\mathbf{G}$ is semisimple, connected and simply connected and if $\tau: \mathbf{G} \rightarrow \mathbf{G}$ is defined over $R$ then, according to a theorem of Raševskii ([Ra1, Ra2]) and Steinberg [St], the complex group $\mathbf{G}(\mathbb{C})^{\tau}=\mathbf{G}^{\tau}(\mathbb{C})$ is connected, so its group of real points $G^{\tau}$ is Zariski connected.

\section{Symplectic Group}

3.1. The symplectic group $G=\operatorname{Sp}(2 n, \mathbb{R})$ may be realized as the group of $2 n$ by $2 n$ real matrices

$$
\begin{aligned}
\mathrm{Sp}(2 n, \mathbb{R}) & =\left\{\left(\begin{array}{ll}
A & B \\
C & D
\end{array}\right) \mid \begin{array}{l}
{ }^{t} A D-{ }^{t} C B=I \\
{ }^{t} A C,{ }^{t} B D \text { symmetric }
\end{array}\right\} \\
& \left.=\left\{\begin{array}{ll}
A & B \\
C & D
\end{array}\right) \mid \begin{array}{l}
A^{t} D-B^{t} C=I \\
A^{t} B, C^{t} D \text { symmetric }
\end{array}\right\}
\end{aligned}
$$


If $J=\left(\begin{array}{cc}0 & I_{n} \\ -I_{n} & 0\end{array}\right)$ is the standard symplectic form on $\mathbb{R}^{2 n}$ then $g \in \mathrm{Sp}(2 n, \mathbb{R})$ iff ${ }^{t} g J g=J$. The inverse of such a symplectic matrix is

$$
\left(\begin{array}{cc}
A & B \\
C & D
\end{array}\right)^{-1}=\left(\begin{array}{cc}
{ }^{t} D & -{ }^{t} B \\
-{ }^{t} C & { }^{t} A
\end{array}\right)
$$

Identify $\mathrm{GL}(n, \mathbb{R}) \hookrightarrow \mathrm{Sp}(2 n, \mathbb{R})$ with its image under the embedding

$$
A \mapsto\left(\begin{array}{cc}
A & 0 \\
0 & A^{-1}
\end{array}\right)
$$

A Cartan involution $\theta$ of $\operatorname{Sp}(2 n, \mathbb{R})$ is given by $\theta g=J g J^{-1}$, that is,

$$
\theta\left(\begin{array}{ll}
A & B \\
C & D
\end{array}\right)=\left(\begin{array}{cc}
D & -C \\
-B & A
\end{array}\right)
$$

Its fixed point set is the unitary group $K=\mathrm{U}(n)$ which is embedded in the symplectic group by

$$
A+i B \mapsto\left(\begin{array}{cc}
A & B \\
-B & A
\end{array}\right) .
$$

The symplectic group acts transitively on the Siegel upper halfspace

$$
\mathfrak{h}_{n}=\left\{Z=X+i Y \in M_{n \times n}(\mathrm{C}) \mid{ }^{t} Z=Z \text { and } Y>0\right\}
$$

by fractional linear transformations: if $g=\left(\begin{array}{ll}A & B \\ C & D\end{array}\right)$ then

$$
g Z=(A Z+B)(C Z+D)^{-1} .
$$

Then $\operatorname{Stab}_{G}(i I)=\mathrm{U}(n)$, and $\mathfrak{h}_{n} \cong G / K$.

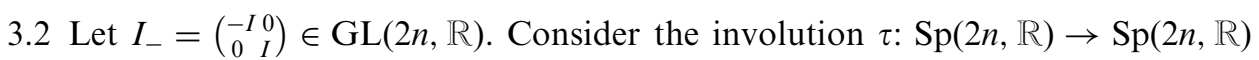
which is defined by $\tau(g)=I_{-} g I_{-}$, that is,

$$
\tau\left(\begin{array}{ll}
A & B \\
C & D
\end{array}\right)=\left(\begin{array}{cc}
A & -B \\
-C & D
\end{array}\right)
$$

The following properties of $\tau$ are easily verified by direct calculation:

(1) $\tau$ is an involution of $G$, i.e. $\tau\left(g h^{-1}\right)=\tau(g)(\tau(h))^{-1}$.

(2) $\tau(g)=g \Leftrightarrow g \in \operatorname{GL}(n, \mathbb{R})$

(3) $\tau \theta=\theta \tau$ so $\tau(\mathrm{U}(n))=\mathrm{U}(n)$

(4) If $C=A+i B \in \mathrm{U}(n)$ then $\tau(C)=\bar{C}=A-i B$.

It follows that $\tau$ passes to an involution (which we denote by the same letter) $\tau: \mathfrak{h}_{n} \rightarrow \mathfrak{h}_{n}$ on the Siegel space such that

$$
\tau(g \cdot Z)=\tau(g) \cdot \tau(Z)
$$

for all $g \in \operatorname{Sp}(2 n, \mathbb{R})$ and for all $Z \in \mathfrak{h}_{n}$. In fact $\tau: \mathfrak{h}_{n} \rightarrow \mathfrak{h}_{n}$ is the antiholomorphic involution given by $\tau(Z)=-\bar{Z}$. Its fixed point set is the orbit

$$
\mathrm{iC}_{n}=\mathrm{GL}(n, \mathbb{R}) \cdot \mathrm{iI} \subset M_{n \times n}(\mathrm{C})
$$


of the general linear group. Here, $C_{n} \cong \mathrm{GL}(n, \mathbb{R}) / \mathrm{O}(n)$ denotes the cone of positive definite symmetric real matrices. (See also Proposition 4.6.)

NOTATION 3.3. For $\gamma \in G$ and $Z \in \mathfrak{h}_{n}$ we will usually write $\bar{\gamma}=\tau(\gamma)$ and $\tilde{Z}=\tau(Z)$. For any $\gamma \in \operatorname{Sp}(2 n, \mathbb{R})$, define the locus of $\gamma$-real points

$$
\mathfrak{h}_{n}^{\tau \gamma}=\left\{Z \in \mathfrak{h}_{n} \mid \gamma Z=-\bar{Z}\right\}
$$

as in Section 2.1. If $\Gamma \subset \operatorname{Sp}(2 n, \mathbb{R})$ is an arithmetic subgroup such that $\tau \Gamma=\Gamma$, define the set $\mathfrak{h}_{n}^{\tau \Gamma}$ of $\Gamma$-real points to be the union $\bigcup_{\gamma \in \Gamma} \mathfrak{h}_{n}^{\tau \gamma}$.

LEMMA 3.4. Let $g \in \mathrm{Sp}(2 n, \mathbb{R})$ and let $\mathfrak{h}_{n}^{g}$ be the set of points in $\mathfrak{h}_{n}$ which are fixed under the action of $g$. Then $\mathfrak{h}_{n}^{g} \cap i C_{n}$ is a proper real algebraic subvariety of $i C_{n}$ unless $g= \pm I \in \mathrm{GL}(n, \mathbb{R})$.

Proof 3.5. Suppose $\left(\begin{array}{ll}A & B \\ C & D\end{array}\right) \cdot i Y=i Y$. Then $A Y=Y D$ and $B=-Y C Y$. These are algebraic equations for $Y$. Suppose every $Y \in C_{n}$ is a solution. Taking $Y=t I$ (with $t>0$ ) gives $A=D$ and $B=-t^{2} C$. So $B=C=0$ and $A Y=Y A$ for all $Y \in C_{n}$, which implies that $A$ is a scalar matrix and that $D={ }^{t} A^{-1}$. So the scalar is \pm 1 .

\section{The Variety $\Gamma(4 m) \backslash \mathfrak{b}_{n}$}

4.1. We shall be concerned with the following arithmetic groups:

- $\Gamma(1)=\operatorname{Sp}(2 n, Z)$

- $\Gamma(N)=\{\gamma \in \Gamma(1) \mid \gamma \equiv I(\bmod N)\}$

- $\Gamma_{2 m}(2)=\left\{\left(\begin{array}{ll}A & B \\ C & D\end{array}\right) \in \Gamma \mid \begin{array}{c}A, D \equiv I(\bmod 2) \\ B, C \equiv 0(\bmod 2 m)\end{array}\right\}$

- $\Gamma_{\ell}(1)=\mathrm{GL}(n, Z)$

- $\Gamma_{\ell}(N)=\left\{\gamma \in \Gamma_{\ell}(1) \mid \gamma \equiv I(\bmod N)\right\}$

(The analogous construction of $\Gamma_{m}(2)$ does not yield a group unless $m$ is even.) Throughout the remainder of the paper we will be interested in the case $N=4 m$ and $m \geqslant 1$. The main result in this section is the following.

THEOREM 4.2. For all $m \geqslant 1$, the mapping $H^{1}(\langle\tau\rangle, \Gamma(4 m)) \rightarrow H^{1}\left(\langle\tau\rangle, \Gamma_{2 m}(2)\right)$ is trivial.

4.3. Let $X=\Gamma(N) \backslash \mathfrak{h}_{n}$. Then $X$ has the structure of a quasi-projective complex algebraic variety. The involution $\tau(Z)=-\bar{Z}$ passes to an anti-holomorphic involution on $X$ and defines a real structure on $X$. (It is a theorem of Shimura [Sh2] that the Baily-Borel compactification $V$ of $X$ even admits the structure of an algebraic variety defined over the rational numbers. See Section 8.) Let $S=\mathfrak{h}_{n}^{\tau \Gamma}$ be the set of all $\Gamma(4 m)$-real points in $\mathfrak{h}_{n}$. Denote by $\Gamma(4 m) \backslash S$ the image of $S$ in $X=\Gamma(4 m) \backslash \mathfrak{h}_{n}$. Note that $\Gamma(4 m)$ is normal in $\Gamma(2)$ and, in the notation of (2.4.1),

$$
\Gamma_{2 m}(2)=\mathcal{G}(\Gamma(4 m), \Gamma(2)) \text {. }
$$

So Lemma 2.5 gives the following: 
COROLLARY 4.4. The set $X_{\mathbb{R}}$ of real points of $X$ is precisely $\Gamma(4 m) \backslash S$. It consists of the disjoint union

$$
X_{\mathrm{R}}=\coprod_{g}{ }^{g} \Gamma_{\ell}(4 m) \backslash g i C_{n}
$$

of finitely many copies of $\Gamma_{\ell}(4 m) \backslash C_{n}$, indexed by elements

$$
g \in \Gamma(4 m) \backslash \Gamma_{2 m}(2) / \Gamma_{\ell}(2)=H^{1}(\langle\tau\rangle, \Gamma(4 m)) .
$$

4.5. The following tower of groups contains $\Gamma(4 m)$ :

$$
\Gamma_{2 m}(2) \subset \Gamma(2) \subset \mathrm{Sp}(2 n, \mathrm{Z}) \subset \mathrm{Sp}(2 n, \mathbb{R}) .
$$

We will show that the image of $H^{1}(\langle\tau\rangle, \Gamma(4 m))$ in the cohomology of each of these is trivial, starting with the rightmost.

PROPOSITION 4.6. With respect to the above action (3.2.1), the (non-Abelian) cohomology sets $H^{1}(\langle\tau\rangle, \mathrm{U}(n))$ and $H^{1}(\langle\tau\rangle, \mathrm{Sp}(2 n, \mathbb{R}))$ are trivial.

Proof 4.7. Let $g=\left(\begin{array}{cc}A & B \\ -B & A\end{array}\right) \in \mathrm{U}(n)$ and suppose $f_{g}$ is a 1-cocycle, that is, $g \tilde{g}=I$. Then $A^{2}+B^{2}=I$ and $A B=B A$. Comparing with (3.1.1) gives ${ }^{t} A=A$ and ${ }^{t} B=B$. Such commuting symmetric matrices may be simultaneously diagonalized by an orthogonal matrix $h \in \mathrm{O}(n)$. In other words, $h(A+i B) h^{-1}=\Lambda \in \mathrm{U}(n)$ is a diagonal matrix. Choose a square root, $\mu=\sqrt{\bar{\Lambda}} \in \mathrm{U}(n)$ (by choosing a square root of each diagonal entry) and set $\delta=h^{-1} \mu h$. Since $\tilde{h}=h$ and $\bar{\mu}=\mu^{-1}$, we find

$$
\tilde{\delta} \delta^{-1}=h^{-1} \tau(\mu) \mu^{-1} h=h^{-1} \bar{\mu} \bar{\mu} h=h^{-1} \Lambda h=A+i B
$$

so $f_{g}$ is a coboundary, hence $H^{1}(\mathrm{U}(n))=0$. From this we may also conclude: if $\gamma \in \mathrm{Sp}(2 n, \mathbb{R})$ and if $f_{\gamma}$ is a 1-cocycle then

$$
f_{\gamma} \text { is a coboundary iff } \mathfrak{h}_{n}^{\tau \gamma} \neq \phi \text {. }
$$

For, suppose $Z \in \mathfrak{h}_{n}^{\tau \gamma}$. Write $Z=h(i I)$ for some $h \in \operatorname{Sp}(2 n, \mathbb{R})$. Then $\tilde{h}^{-1} \gamma h(i I)=$ $\tilde{h}^{-1} \gamma Z=\tilde{h}^{-1} \tilde{Z}=\tau(i I)=i I$ hence $\alpha=\tilde{h}^{-1} \gamma h \in \mathrm{U}(n)$. Moreover, $\alpha \tilde{\alpha}=\tilde{h}^{-1} \gamma \tilde{\gamma} \tilde{h}=I$ so by the preceding paragraph there exists $\delta \in \mathrm{U}(n)$ with $\alpha=\tilde{\delta} \delta^{-1}$. Therefore $\gamma=\tau(h \delta)(h \delta)^{-1}$.

At this point we remark that if $\gamma=\left(\begin{array}{ll}A & B \\ C & D\end{array}\right) \in \mathrm{Sp}(2 n, \mathbb{R})$ and if $\gamma \tilde{\gamma}=I$, then

$$
A={ }^{t} D, C={ }^{t} C, B={ }^{t} B, A^{2}-B C=I, C A={ }^{t} A C \text {, and } A B=B{ }^{t} A \text {. }
$$

Next we consider two special cases. Let $\gamma=\left(\begin{array}{cc}A & B \\ C & D\end{array}\right) \in \operatorname{Sp}(2 n, \mathbb{R})$ and suppose $\gamma \tilde{\gamma}=I$. Suppose either (1) $A=D=0$ and $C=-B^{-1}$ is diagonal, or (2) $C=0$. Then $f_{\gamma}$ is a coboundary. For in case (1), let $B=\operatorname{diag}\left(b_{1}, b_{2}, \ldots, b_{n}\right)$ and let $Z=i Y$ where $Y=\operatorname{diag}\left(\left|b_{1}\right|,\left|b_{2}\right|, \ldots,\left|b_{n}\right|\right)$. Then $\gamma \cdot Z=-B Z^{-1} B=-\bar{Z}=\tau(Z)$ so $f_{\gamma}$ is a coboundary by (4.7.1). In case (2), the cocycle $f_{\gamma}$ is equivalent to $f_{\gamma^{\prime}}$ where

$$
\gamma^{\prime}=\left(\begin{array}{cc}
A & 0 \\
0 & { }^{t} A
\end{array}\right)=\tilde{h} \gamma h^{-1}
$$


and

$$
h=\left(\begin{array}{cc}
I & \frac{1}{2} A^{-1} B \\
0 & I
\end{array}\right)
$$

using (4.7.2). Then $A^{2}=I$ so $A$ is $\operatorname{GL}(n, \mathbb{R})$-conjugate to a diagonal matrix $\Lambda$ (with eigenvalues \pm 1$)$. Therefore $f_{\gamma^{\prime}}$ is equivalent to the 1 -cocycle defined by the element $\left(\begin{array}{cc}\Lambda & 0 \\ 0 & \Lambda^{-1}\end{array}\right) \in \mathrm{U}(n)$. It follows that $f_{\gamma^{\prime}}$ is cohomologically trivial.

Now consider the case of a general 1-cocycle $f_{\gamma^{\prime}}$ where $\gamma^{\prime}=\left(\begin{array}{ll}A^{\prime} & B^{\prime} \\ C^{\prime} & D^{\prime}\end{array}\right)$. By (4.7.2), $C^{\prime}$ is symmetric so there exists $k \in \mathrm{O}(n)$ so that $C=k C^{\prime t} k=\left(\begin{array}{cc}C_{1} & 0 \\ 0 & 0\end{array}\right)$ where $C_{1} \in M_{r \times r}(\mathbb{R})$ is diagonal and nonsingular. Let $h_{1}=\left(\begin{array}{ll}k & 0 \\ 0 & k\end{array}\right)$. Then $f_{\gamma^{\prime}}$ is cohomologous to $f_{\gamma}$ where

$$
\gamma=\tilde{h}_{1} \gamma^{\prime} h_{1}^{-1}=\left(\begin{array}{cc}
A & B \\
C & D
\end{array}\right)
$$

and (4.7.2) holds. Write $A=\left(\begin{array}{ll}A_{1} & A_{2} \\ A_{4} & A_{4}\end{array}\right)$ where $A_{1} \in M_{r \times r}(\mathbb{R})$ and $A_{4} \in M_{(n-r) \times(n-r)}(\mathbb{R})$, and similarly for $B$ and $D$. Then $C A={ }^{t} A C$ implies that $A_{2}=A_{3}=0$.

Set $h_{2}=\left(\begin{array}{ll}I & x \\ 0 & I\end{array}\right)$ where

$$
x=\left(\begin{array}{ccc}
C_{1}^{-1}{ }^{t} A_{1} & 0 \\
0 & 0
\end{array}\right) .
$$

Then $A^{2}-B C=I$ implies that $f_{\gamma}$ is cohomologous to $f_{g}$ where

$$
g=\bar{h}_{2} \gamma h_{2}^{-1}=\left(\begin{array}{c|c}
A-x C & -A x+B+x C_{x}-x^{t} A \\
\hline C & -C x+{ }^{t} A
\end{array}\right)=\left(\begin{array}{cc|cc}
0 & 0 & -C_{1}^{-1} & 0 \\
0 & A_{4} & 0 & B_{4} \\
\hline C_{1} & 0 & 0 & 0 \\
0 & 0 & 0 & { }^{t} A_{4}
\end{array}\right) .
$$

Since $g \tilde{g}=I$ use case (1) and (2) above to find $Z_{r} \in \mathfrak{h}_{r}$ and $Z_{n-r} \in \mathfrak{h}_{n-r}$ so that

$$
\left(\begin{array}{cc}
0 & -C_{1}^{-1} \\
C_{1} & 0
\end{array}\right) Z_{r}=-\bar{Z}_{r} \quad \text { and } \quad\left(\begin{array}{cc}
A_{4} & B_{4} \\
0 & { }^{t} A_{4}
\end{array}\right) Z_{n-r}=-\bar{Z}_{n-r} .
$$

Set $Z=\left(\begin{array}{lr}Z_{r} & 0 \\ 0 & Z_{n-r}\end{array}\right)$. Then $g Z=-\bar{Z}$ hence the corresponding cocycle is cohomologically trivial.

LEMMA 4.8. Let $g \in \mathrm{Sp}(2 n, Z)$. Fix $m \geqslant 1$.

(1) If $g \in \Gamma(m)$ then $\tilde{g} g^{-1} \in \Gamma(2 m)$.

(2) If $g \in \Gamma(2 m)$ then $\tilde{g} g \in \Gamma(4 m)$.

(3) If $\tilde{g} g^{-1} \in \Gamma(4 m)$ then $g=\beta u$ for some $\beta \in \Gamma_{2 m}(2)$ and for some $u \in \mathrm{GL}(n, \mathbb{Z})$.

Proof 4.9. Let $g=\left(\begin{array}{ll}A & B \\ C & D\end{array}\right)$. Since $\tilde{g}=g-\left(\begin{array}{cc}0 & 2 B \\ 2 C & 0\end{array}\right)$ we have

$$
\tilde{g} g^{-1}=I-\left(\begin{array}{cc}
-2 B^{t} C & 2 B^{t} A \\
2 C^{t} D & -2 C^{t} A
\end{array}\right) \text {. }
$$


This gives part (1). If $g=I+2 m g^{\prime}$ then $\tilde{g}+g$ is even, and $\tilde{g} g=I+2 m\left(\tilde{g}^{\prime}+g^{\prime}\right)+$ $4 m^{2} \tilde{g}^{\prime} g$ which proves part (2). Now suppose that $\tilde{g} g^{-1} \in \Gamma(4 m)$ so that $B{ }^{t} C \equiv$ $\left.B^{t} A \equiv C^{t} D \equiv 0 \bmod 2 m\right)$. Then

$$
\begin{aligned}
B & =B\left({ }^{t} A D-{ }^{t} C B\right)=\left(B{ }^{t} A\right) D-\left(B{ }^{t} C\right) B \equiv 0(\bmod 2 m) \\
{ }^{t} C & =\left({ }^{t} A D-{ }^{t} C B\right){ }^{t} C={ }^{t} A\left(D{ }^{t} C\right)-{ }^{t} C\left(B{ }^{t} C\right) \equiv 0(\bmod 2 m) .
\end{aligned}
$$

Moreover, since $C^{t} B \equiv 0(\bmod (2 m))$ and $A^{t} D-B{ }^{t} C=I$ we see that $A^{t} D \equiv$ $I(\bmod 2)$. Hence $A$ is invertible $(\bmod 2)$. But reduction $(\bmod 2)$ is a surjective mapping $\operatorname{GL}(n, Z) \rightarrow \operatorname{GL}(n, Z /(2))$ hence there exists $U \in \mathrm{GL}(n, Z)$ so that $U \equiv$ $A(\bmod 2)$ from which it also follows that $U^{-1} \equiv{ }^{t} D(\bmod 2)$. Let $u=$ $\left(\begin{array}{cc}U & 0 \\ 0^{t} U^{-1}\end{array}\right)$. Then

$$
\beta=g u^{-1}=\left(\begin{array}{ll}
A U^{-1} & B^{t} U \\
C U^{-1} & D^{t} U
\end{array}\right) \in \Gamma_{2 m}(2) .
$$

The following proposition is a consequence of the theorem of Silhol [Si] (Theorem 1.4) and Comessatti, and will be proven in Section 7.

PROPOSITION 4.10. Let $\gamma \in \Gamma(2)$ and suppose that $Z \in \mathfrak{h}_{n}$ is not fixed by any element of $\operatorname{Sp}(2 n, Z)$ other than $\pm I$. Suppose that $\tilde{Z}=\gamma Z$. Then there exists $h \in \operatorname{Sp}(2 n, Z)$ such that $\gamma=\tilde{h} h^{-1}$.

\subsection{PROOF OF THEOREM 4.2.}

Let $\gamma \in \Gamma(4 m)$ and suppose $f_{\gamma}$ is a cocycle. Its image in $H^{1}(\langle\tau\rangle, \operatorname{Sp}(2 n, \mathbb{R}))$ is a coboundary so there exists $h \in \operatorname{Sp}(2 n, \mathbb{R})$ with $\gamma=\tilde{h} h^{-1}$. This implies that $\mathfrak{h}_{n}^{\tau \gamma}=h i C_{n}$ is a copy of the cone of positive definite matrices. By Lemma 3.4 there exist points $Z \in \mathfrak{h}_{n}^{\tau \gamma}$ which are not fixed by any element of $\operatorname{Sp}(2 n, Z)$ other than $\pm I$ and in fact the set of such points is the complement of a countable union of proper (real) algebraic subvarieties of $\mathfrak{h}_{n}^{\tau \gamma}$. Applying Proposition 4.10, we conclude that $\gamma=\tilde{h} h^{-1}$ for some $h \in \operatorname{Sp}(2 n, Z)$. By Lemma 4.8 (3) we may write $h=\beta u$ for some $\beta \in \Gamma_{2 m}(2)$ and some $u \in \operatorname{GL}(n, Z)$. Then $\tilde{h} h^{-1}=\tilde{\beta} \beta^{-1}=\gamma$ which proves that $f_{\gamma}$ is trivial in $H^{1}\left(\langle\tau\rangle, \Gamma_{2 m}(2)\right)$.

\section{Upper Half-plane}

For the upper half-plane $\mathfrak{h}_{1}$ it is possible to do a little better since the group $\Gamma(2) / \pm I$ acts freely.

LEMMA 5.1. A point $Z \in \mathfrak{h}_{1}$ is $\Gamma(2)$-real if and only if $g Z$ is $\Gamma(2)$-real, for every $g \in \operatorname{SL}(2, Z)$.

Proof 5.2. Suppose $\tau(Z)=\gamma Z$ for some $\gamma \in \Gamma(2)$. Then

$$
\tau(g z)=\tau(g) \tau(z)=\left(\tau(g) g^{-1}\right)\left(g \gamma g^{-1}\right) g Z \in \Gamma(2) \cdot \Gamma(2) \cdot g Z .
$$


LEMMA 5.3. If a point $y \in \mathfrak{h}_{1}$ is $\Gamma(2)$-real, then there exists $\beta \in \mathrm{SL}(2, Z)$ and there exists $Y \in C_{1}=\mathbb{R}_{+}$so that $y=\beta \cdot i Y$.

Proof 5.4. Say $\tilde{y}=\gamma y$ for some $\gamma \in \Gamma(2)$. We consider two cases: (a) when $y$ is not fixed by any element of $\mathrm{SL}_{2}(Z)$ other than $\pm I$ and (b) when $y$ is fixed by some other nontrivial element of $\mathrm{SL}_{2}(\mathrm{Z})$.

In case (a), the result is just Proposition 4.10. Now consider case (b). There are two classes of elements which are fixed by nontrivial subgroups of $\mathrm{SL}_{2}(\mathrm{Z})$. These are the translates (by elements of $\mathrm{SL}_{2}(Z)$ ) of $i$ and the translates of $\omega=\mathrm{e}^{2 \pi i / 3}$. In the first case, $y=\beta \cdot i$ (for some $\beta \in \mathrm{SL}_{2}(Z)$ ) and the conclusion follows. In the second case, the stabilizer of $\omega$ is the subgroup

$$
S=\left\{ \pm I, \pm\left(\begin{array}{rr}
0 & -1 \\
1 & 1
\end{array}\right), \pm\left(\begin{array}{rr}
-1 & -1 \\
1 & 0
\end{array}\right)\right\}
$$

Suppose $y=g \omega$ (for some $g \in \mathrm{SL}_{2}(Z)$ ). We claim that $y$ is not $\Gamma(2)$-real. Suppose otherwise. Then $\tilde{y}=\gamma y$ gives $\tilde{\omega}=\gamma^{\prime} \omega$ where

$$
\gamma^{\prime}=\tilde{g}^{-1} \gamma g=\left(\tilde{g}^{-1} g\right) g^{-1} \gamma g \in \Gamma(2)
$$

by Lemma 4.8. But $\tilde{\omega}=-\bar{\omega}=j \omega$ where $j=\left(\begin{array}{cc}0 & -1 \\ 1 & 0\end{array}\right)$. So $j^{-1} \gamma^{\prime}$ stabilizes $\omega$ or $\gamma^{\prime}=j s$ for some $s \in S$. However none of these matrices $j s$ lies in $\Gamma(2)$, which is a contradiction. This completes the proof of Lemma 5.3.

The group $\Gamma(2)$ does not induce any identifications on the cone $i C_{1}$. However, $i C_{1}$ is preserved by the subgroup $S^{\prime}=\{ \pm I, \pm v\}$ where $v=\left(\begin{array}{cc}0 & -1 \\ 1 & 0\end{array}\right)$. So we obtain

THEOREM 5.5. The set of real points of $X=\Gamma(2) \backslash \mathfrak{G}_{1}$ consists of the union of 3 copies of the cone $C_{1} \cong \mathbb{R}_{+}$,

$$
X_{\mathrm{R}}=\bigcup_{\beta \in \Gamma(2)\left(\mathrm{SL}_{2} \mathrm{Z}\right) / S^{\prime}} \beta \cdot i C_{1}
$$

We remark that a fundamental domain for $X_{\mathbb{R}}$ in $\mathfrak{h}_{1}$ is the union of the half lines with real part 0 and 1 and the semicircle of radius $1 / 2$ centered at $1 / 2$. In the Baily-Borel compactification of $X$ these three half lines are joined end to end, to form a single circle which passes through the three distinct cusps $\infty, 0,1$.

\section{Moduli Space Interpretation}

6.1. Recall [La] that a symplectic form $Q$ on $\mathrm{C}^{n}$ is compatible with the complex structure if $Q(i u, i v)=Q(u, v)$ for all $u, v \in \mathbb{C}^{n}$. A compatible form $Q$ is positive if the symmetric form $R(u, v)=Q(i u, v)$ is positive definite. If $Q$ is compatible and positive then it is the imaginary part of a unique positive definite Hermitian form $H=R+i Q$. Let $L \subset \mathbb{C}^{n}$ be a lattice and let $H=R+i Q$ be a positive definite Hermitian form on $\mathbb{C}^{n}$. 
A basis of $L$ is symplectic if the matrix for $Q$ with respect to this basis is $\left(\begin{array}{cc}0 & I \\ -I & 0\end{array}\right)$. The lattice $L$ is symplectic if it admits a symplectic basis.

A principally polarized Abelian variety is a pair $\left(A=\mathbb{C}^{n} / L, H=R+i Q\right)$ where $H$ is a positive definite Hermitian form on $\mathbb{C}^{n}$ and where $L \subset \mathbb{C}^{n}$ is a symplectic lattice relative to $Q=\operatorname{Im}(H)$. A real structure on $(A, H)$ is a complex anti-linear involution $\kappa: \mathbb{C}^{n} \rightarrow \mathbb{C}^{n}$ such that $\kappa(L)=L$. A real structure $\kappa$ on $(A, H)$ is compatible with the polarization $H$ if $Q(\kappa u, \kappa v)=-Q(u, v)$ for all $u, v \in \mathbb{C}^{n}$. In this case (following [Si]) we refer to the triple $(A, H, \kappa)$ as a real principally polarized Abelian variety. If $\left(A^{\prime}, H^{\prime}, \kappa^{\prime}\right)$ is another such, then an isomorphism between them is a complex linear mapping $\phi: \mathbb{C}^{n} \rightarrow \mathbb{C}^{n}$ such that

(a) $\phi(L)=L^{\prime}$,

(b) $\phi_{*}(H)=H^{\prime}$,

(c) $\phi_{*}(\kappa)=\kappa^{\prime}$, that is, $\phi \kappa \phi^{-1}=\kappa^{\prime}$.

Let $\left(A=\mathrm{C}^{n} / L, H=R+i Q\right)$ be a principally polarized Abelian variety. A level $N$ structure on $A$ is a choice of basis $\left\{U_{i}, V_{j}\right\}$ (with $1 \leqslant i, j \leqslant n$ ) for the $N$-torsion points of $A$, which is symplectic, in the sense that there exists a symplectic basis $\left\{u_{i}, v_{j}\right\}$ for $L$ such that

$$
U_{i} \equiv \frac{u_{i}}{N} \quad \text { and } \quad V_{j} \equiv \frac{v_{j}}{N}(\bmod L)
$$

(for $1 \leqslant i, j \leqslant n$ ). For a given leven $N$ structure, such a choice $\left\{u_{i}, v_{j}\right\}$ determines a mapping

$$
F: \mathbb{R}^{n} \oplus \mathbb{R}^{n} \rightarrow \mathbb{C}^{n}
$$

such that $F\left(Z^{n} \oplus Z^{n}\right)=L$, by $F\left(e_{i}\right)=u_{i}$ and $F\left(f_{j}\right)=v_{j}$ where $\left\{e_{i}, f_{j}\right\}$ (with $1 \leqslant i, j$ $\leqslant n$ ) is the standard basis of $\mathbb{R}^{n} \oplus \mathbb{R}^{n}$. The choice $\left\{u_{i}, v_{j}\right\}$ (or equivalently, the mapping $F$ ) will be referred to as a lift of the level $N$ structure. It is well defined modulo the principal congruence subgroup $\Gamma(N)$, that is, if $F^{\prime}: \mathbb{R}^{n} \oplus \mathbb{R}^{n} \rightarrow \mathbb{C}^{n}$ is another lift of the level structure, then $F^{\prime} \circ F^{-1} \in \Gamma(N)$.

A level $N$-structure $\left\{U_{i}, V_{j}\right\}$ is compatible with a real structure $\kappa$ if for some (and hence for any) lift $\left\{u_{i}, v_{j}\right\}$ of the level structure,

$$
\kappa\left(\frac{u_{i}}{N}\right) \equiv-\frac{u_{i}}{N}(\bmod L) \quad \text { and } \quad \kappa\left(\frac{v_{j}}{N}\right) \equiv+\frac{v_{j}}{N}(\bmod L)
$$

for all $1 \leqslant i, j \leqslant n$. In other words, the following diagram commutes $(\bmod L)$.

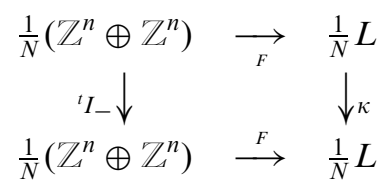

where (cf. Section 3.2) $I_{-}=\left(\begin{array}{cc}-I & 0 \\ 0 & I\end{array}\right)$ 
DEFINITION 6.2. A real principally polarized Abelian variety with level $N$ structure is a quadruple $\mathcal{A}=\left(A=\mathrm{C}^{n} / L, H=R+i Q, \kappa,\left\{U_{i}, V_{j}\right\}\right)$ where $(A, H, \kappa)$ is a real principally polarized Abelian variety and where $\left\{U_{i}, V_{j}\right\}$ is a level $N$-structure which is compatible with $\kappa$. An isomorphism

$$
\mathcal{A}=\left(A, H, \kappa,\left\{U_{i}, V_{j}\right\}\right) \cong\left(A^{\prime}, H^{\prime}, \kappa^{\prime},\left\{U_{i}^{\prime}, V_{j}^{\prime}\right\}\right)=\mathcal{A}^{\prime}
$$

is a complex linear mapping $\phi: \mathbb{C}^{n} \rightarrow \mathbb{C}^{n}$ such that (a), (b), and (c) of Section 6.1 hold, and such that

(d) $\phi\left(\frac{u_{i}}{N}\right) \equiv \frac{u_{i}^{\prime}}{N}\left(\bmod L^{\prime}\right)$ and $\phi\left(\frac{v_{j}}{N}\right) \equiv \frac{v_{j}^{\prime}}{N}\left(\bmod L^{\prime}\right)$ for $1 \leqslant i, j \leqslant n$

for some (and hence for any) lift $\left\{u_{i}, v_{j}\right\}$ and $\left\{u_{i}^{\prime}, v_{j}^{\prime}\right\}$ of the level structures.

6.3. Fix $N \geqslant 1$. Each $Z \in \mathfrak{h}_{n}$ determines a principally polarized Abelian variety $\left(A_{Z}, H_{Z}\right)$ with level $N$ structure as follows. Let $Q_{0}$ be the standard symplectic form

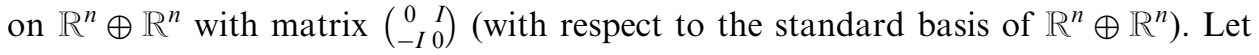
$F_{Z}: \mathbb{R}^{n} \oplus \mathbb{R}^{n} \rightarrow \mathbb{C}^{n}$ be the real linear mapping with matrix $(Z, I)$, that is,

$$
F_{Z}\left(\begin{array}{l}
x \\
y
\end{array}\right)=Z x+y
$$

Then $Q_{Z}=\left(F_{Z}\right)_{*}\left(Q_{0}\right)$ is a compatible, positive symplectic form and $L_{Z}=F_{Z}$ $\left(Z^{n} \oplus Z^{n}\right)$ is a symplectic lattice with symplectic basis $F_{Z}$ (standard basis). The Hermitian form corresponding to $Q_{Z}$ is

$$
H_{Z}(u, v)=Q_{Z}(i u, v)+i Q_{Z}(u, v)={ }^{t} u(\operatorname{Im}(Z))^{-1} \bar{v} \quad \text { for } u, v \in \mathbb{C}^{n} .
$$

Then the pair $\left(A_{Z}=\mathbb{C}^{n} / L_{Z}, H_{Z}\right)$ is a principally polarized Abelian variety. If $z_{1}, z_{2}$ are the standard coordinates on $\mathbb{C}^{n}$ then, with respect to the above symplectic basis of $L$, the differential forms $\mathrm{d} z_{1}, \mathrm{~d} z_{2}$ have period matrix $(Z, I)$. If $\left\{e_{1}, \ldots, e_{n}\right.$, $\left.f_{1}, \ldots, f_{n}\right\}$ denote the standard basis elements of $\mathbb{R}^{n} \oplus \mathbb{R}^{n}$ then the collection $\left\{F_{Z}\left(e_{i} / N\right), F_{Z}\left(f_{i} / N\right)\right\}(\bmod L)$ is a level $N$ structure on $\left(A_{Z}, H_{Z}\right)$, which we refer to as the standard level $N$ structure.

Let $Z, \Omega \in \mathfrak{h}_{n}$. Suppose $\psi:\left(A_{\Omega}=\mathbb{C}^{n} / L_{\Omega}, H_{\Omega}\right) \rightarrow\left(A_{Z}=\mathbb{C}^{n} / L_{Z}, H_{Z}\right)$ is an isomorphism of the corresponding principally polarized Abelian varieties, that is, $\psi\left(L_{\Omega}\right)=L_{Z}$ and $\psi_{*}\left(H_{\Omega}\right)=H_{Z}$. Set $h={ }^{t}\left(F_{Z}^{-1} \psi F_{\Omega}\right)=\left(\begin{array}{cc}A & B \\ C & D\end{array}\right)$. Then: $h \in \operatorname{Sp}(2 n, Z)$, $\Omega=h \cdot Z$, and $\psi(M)={ }^{t}(C Z+D) M$ for all $M \in \mathbb{C}^{n}$. Since $h \cdot Z$ is symmetric, the following diagram commutes:

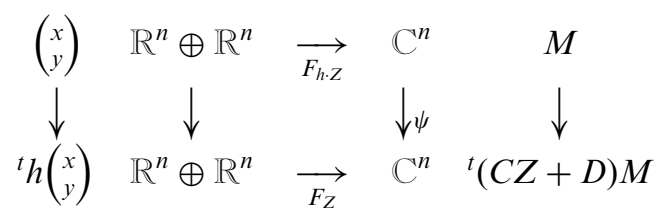


Let $Z \in \mathfrak{h}_{n}$ with $\tilde{Z}=\tau(Z)=-\bar{Z}$. Then the following diagram commutes:

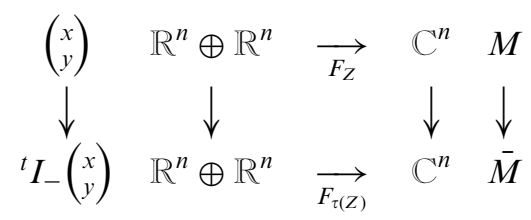

6.4. The points in $\mathfrak{G}_{n}^{\tau \Gamma(1)}$ give rise to Abelian varieties with a real structure, as follows. Suppose $Z \in \mathfrak{h}_{n}, \gamma=\left(\begin{array}{ll}A & B \\ C & D\end{array}\right) \in \mathrm{Sp}(2 n, Z)$ and suppose that $\gamma \cdot Z=\tau(Z)$. Define

$$
\kappa(\gamma, Z): \mathbb{C}^{n} \rightarrow \mathbb{C}^{n} \text { by } M \mapsto{ }^{t}(C Z+D) \bar{M} .
$$

Then $\kappa(\gamma, Z)$ is a real structure on $\left(A_{Z}, H_{Z}\right)$ which is compatible with the polarization $H_{Z}$, and the following diagram (which is the composition of the two preceding diagrams) commutes:

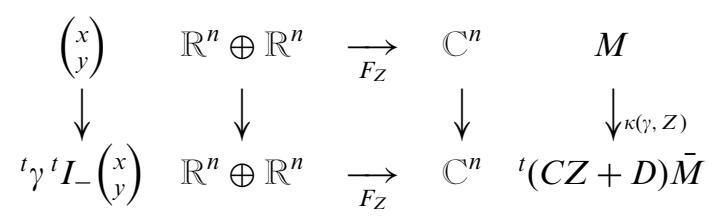

PROPOSITION 6.5. Let $Z \in \mathfrak{h}_{n}$ and $\gamma \in \mathrm{Sp}(2 n, \mathbb{R})$ and suppose that $\tilde{Z}=\gamma \cdot Z$. Then $\gamma \in \Gamma(N)$ iff the real structure $\kappa(\gamma, Z)$ on $\left(A_{Z}, H_{Z}\right)$ is compatible with the standard level $N$ structure, and in this case the quadruple

$$
\left(A_{Z}, H_{Z}, \kappa(\gamma, Z),\left\{F_{Z}\left(e_{i} / N\right), F_{Z}\left(f_{j} / N\right)\right\}\right)
$$

is a real principally polarized abelian variety with (compatible) level $N$ structure.

The proof follows immediately from the diagrams (6.4.2) and (6.1.2). We remark that if $N \geqslant 3$, and if $Z \in \mathfrak{h}_{n}^{\tau \Gamma(N)}$ then there is a unique $\gamma \in \Gamma(N)$ such that $\tilde{Z}=\gamma \cdot Z$ so we may unambiguously denote $\kappa(\gamma, Z)$ simply by $\kappa_{Z}$. In this case, denote by $\mathcal{A}_{Z}$ the resulting real Abelian variety with principal polarization and (compatible) level $N$ structure. The proof of the following theorem will appear in Section 7.

THEOREM 6.6. Fix $N=4 m$ with $m \geqslant 1$. The association $Z \mapsto \mathcal{A}_{Z}$ determines a one to one correspondence between the points of $X_{\mathbb{R}}$ and the set of isomorphism classes of real principally polarized Abelian varieties with level $N$ structure.

\section{The Comessatti Lemma}

Recall the following theorem of Silhol [Si] Theorem 1.4., (also [Si2]) and Comessatti [C]. 
THEOREM 7.1. Let $(A, H, \kappa)$ be a real principally polarized Abelian variety. Then there exists $Z=X+i Y \in \mathfrak{h}_{n}$ so that $2 X$ is integral, and there exists an isomorphism of real principally polarized Abelian varieties,

$$
(A, H, \kappa) \cong\left(A_{Z}, H_{Z}, \sigma\right)
$$

where $\sigma(M)=\bar{M}$ is complex conjugation.

For the purposes of this paper we will need a slight restatement of this result.

LEMMA 7.2. (A) Let $Z \in \mathfrak{h}_{n}$ and suppose that $\tilde{Z}=\gamma \cdot Z$ for some $\gamma \in \operatorname{Sp}(2 n, Z)$. Then $Z$ is equivalent under $\operatorname{Sp}(2 n, Z)$ to an element $X+i Y \in \mathfrak{h}_{n}$ such that $2 X$ is integral.

(B) Let $(A, H, \kappa)$ be a real principally polarized Abelian variety. Then there exists $\gamma \in \operatorname{Sp}(2 n, Z)$ and there exists $Z=X+i Y \in \mathfrak{h}_{n}$ such that $\tilde{Z}=\gamma \cdot Z$ and $2 X$ is integral; and there exists an isomorphism of real principally polarized Abelian varieties

$$
(A, H, \kappa) \cong\left(A_{Z}, H_{Z}, \kappa(\gamma, Z)\right) \text {. }
$$

Proof 7.3. For part (B), Theorem 7.1 provides $Z=X+i Y \in \mathfrak{h}_{n}$ so that $2 X$ is integral. Take $\gamma=\left(\begin{array}{cc}I & -2 X \\ 0 & I\end{array}\right)$. Then $\tilde{Z}=-\bar{Z}=\gamma \cdot Z$ and $\kappa(\gamma, Z)(M)=\bar{M}=\sigma(M)$ by (6.4.2).

For part (A), given $Z$ and $\gamma$, apply part (B) to the real Abelian variety $\left(A_{Z}, H_{Z}, \kappa(\gamma, Z)\right)$ to obtain an isomorphic real Abelian variety $\left(A_{Z^{\prime}}, H_{Z^{\prime}}, \kappa\left(\gamma^{\prime}, Z^{\prime}\right)\right)$ such that $Z^{\prime}=X^{\prime}+i Y^{\prime}$ with $2 X^{\prime}$ integral. Then $\left(A_{Z}, H_{Z}\right)$ and $\left(A_{Z^{\prime}}, H_{Z^{\prime}}\right)$ are isomorphic principally polarized Abelian varieties so there exists $g \in \operatorname{Sp}(2 n, Z)$ with $Z^{\prime}=g \cdot Z$.

\subsection{PROOF OF PROPOSITION 4.10.}

By Comessatti's lemma, $Z$ is equivalent (via some $h \in \operatorname{Sp}(2 n, Z)$ ) to some element $X+i Y \in \mathfrak{h}_{n}$ with $2 X \in M_{n \times n}(Z)$. We claim, in this case, that $X$ is integral. Translation by $X$ is given by the symplectic matrix

$$
T_{X}=\left(\begin{array}{cc}
I & X \\
0 & I
\end{array}\right)
$$

so we may write $Z=h(X+i Y)=h T_{X} i Y$. Then $\tau Z=\tau\left(h T_{X}\right) i Y=\gamma Z$, so the following element $\gamma^{-1} \tau\left(h T_{X}\right)\left(h T_{X}\right)^{-1}$ fixes $Z$. By our assumption on $Z$, this implies that

$$
\gamma= \pm I \tau\left(h T_{X}\right)\left(h T_{X}\right)^{-1}
$$

or,

$$
\pm T_{-2 X}=\tilde{h}^{-1} \gamma h=\left(\tilde{h}^{-1} h\right)\left(h^{-1} \gamma h\right) \in \Gamma(2) . \Gamma(2)
$$

(using Lemma 4.8 and the fact that $\Gamma(2)$ is normal in $\mathrm{Sp}(2 n, Z)$ ). So $2 X$ is 'even', hence $X$ is integral. If the plus sign occurs in (7.4.1) then $\gamma=\tau\left(h T_{X}\right)\left(h T_{X}\right)^{-1}$. If the minus sign occurs, set $\omega=\left(\begin{array}{cc}0 & -I \\ I & 0\end{array}\right)$. Then $\gamma=\tau\left(h T_{X} \omega\right)\left(h T_{X} \omega\right)^{-1}$.

In the next proposition we strengthen these results to include level structures. 
PROPOSITION 7.5. Suppose $N \geqslant 3$. Suppose $\mathcal{A}$ is a real Abelian variety with principal polarization and level $N$ structure. Then there exists $Z \in \mathfrak{h}_{n}$, there exists $\gamma \in$ $\Gamma(N)$ such that $\gamma \cdot Z=\tau(Z)$, and there exists an isomorphism $\phi: \mathcal{A}_{Z} \rightarrow \mathcal{A}$ of real principally polarized Abelian varieties with level structures. If $N=4 m$ (and $m \geqslant 1)$ the cohomology class $\left[f_{\gamma}\right] \in H^{1}(\langle\tau\rangle, \Gamma(4 m))$ is uniquely determined by the isomorphism class of $\mathcal{A}$.

Proof 7.6. Write $\mathcal{A}=\left(A=\mathrm{C}^{n} / L, H=R+i Q, \kappa,\left\{U_{i}, V_{j}\right\}\right)$. By Lemma 7.2, there exists $Z^{\prime} \in \mathfrak{h}_{n}$, there exists $\gamma^{\prime} \in \mathrm{Sp}(2 n, Z)$ with $\gamma^{\prime} \cdot Z^{\prime}=\tau\left(Z^{\prime}\right)$, and there exists $\phi^{\prime}: \mathbb{C}^{n} \rightarrow \mathbb{C}^{n}$ such that $\phi^{\prime}$ induces an isomorphism $(A, H, \kappa) \cong\left(A_{Z^{\prime}}, H_{Z^{\prime}}, \kappa\left(\gamma^{\prime}, Z^{\prime}\right)\right)$, that is:

(a) $\phi^{\prime}\left(L_{Z^{\prime}}\right)=L$

(b) $\phi_{*}^{\prime}\left(H_{Z^{\prime}}\right)=H$

(c) $\phi_{*}^{\prime}\left(\kappa\left(\gamma^{\prime}, Z^{\prime}\right)\right)=\kappa$.

The isomorphism $\phi^{\prime}$ must be modified because it does not necessarily take the standard level $N$ structure on $\left(A_{Z^{\prime}}, H_{Z^{\prime}}\right)$ to the given level $N$ structure on $(A, H)$. Choose a lift $\left\{u_{i}, v_{j}\right\}(1 \leqslant i, j \leqslant n)$ of the level $N$ structure on $(A, H)$ and let $F: \mathbb{R}^{2} \oplus \mathbb{R}^{2} \rightarrow \mathbb{C}^{2}$ be the resulting mapping (6.1.1). Define

$$
\begin{aligned}
& { }^{t} g^{-1}=F^{-1} \circ \phi^{\prime} \circ F_{Z^{\prime}} \in \operatorname{Sp}(2 n, Z) \\
& Z=g \cdot Z^{\prime} \\
& \gamma=\tau(g) \gamma^{\prime} g^{-1}=I_{-} g I_{-} \gamma^{\prime} g^{-1} .
\end{aligned}
$$

If $g=\left(\begin{array}{ll}A & B \\ C & D\end{array}\right)$ define $\psi: \mathbb{C}^{n} \rightarrow \mathbb{C}^{n}$ by $\psi(M)={ }^{t}(C Z+D) M$. We claim that $\gamma \in \Gamma(N)$, that $\tilde{Z}=\gamma \cdot Z$ and that the mapping

$$
\phi=\phi^{\prime} \circ \psi: \mathbb{C}^{n} \rightarrow \mathbb{C}^{n}
$$

induces an isomorphism $\mathcal{A}_{Z} \cong \mathcal{A}$ between principally polarized real Abelian varieties with (compatible) level $N$ structures.

The matrix $g$ is defined so that the bottom square in the following diagram commutes; by (6.3.1) the top square also commutes. The mapping $\phi$ is the composition down the right-hand column.

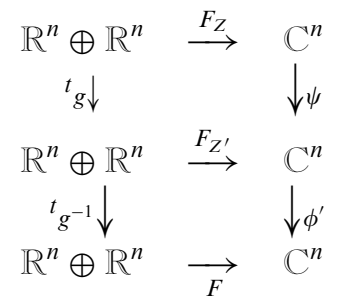

Clearly, $\tilde{Z}=\gamma \cdot Z, \phi_{*}\left(L_{Z}\right)=L$ and $\phi_{*}\left(H_{Z}\right)=H$. Now let us check that

$$
\phi_{*}(\kappa(\gamma, Z))=\kappa .
$$


By Section 7.6 (c) it suffices to check that $\psi \kappa(\gamma, Z) \psi^{-1}=\kappa\left(\gamma^{\prime}, Z^{\prime}\right)$. But this follows

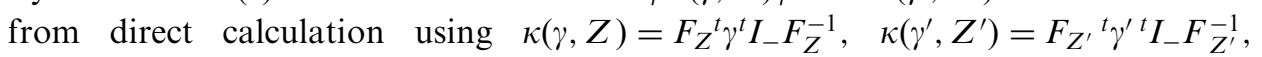
$\psi=F_{Z^{\prime}}{ }^{t} g F_{Z}^{-1}$, and (7.6.3).

By (7.6.4), $F=\phi \circ F_{Z}$. Therefore $\phi$ preserves the level structures. Since the given level structure on $(A, H)$ is compatible with $\kappa$, equation (7.6.5) guarantees that the standard level $N$ structure on $\left(A_{Z}, H_{Z}\right)$ is compatible with $\kappa(\gamma, Z)$. It follows from Proposition 6.5 that $\gamma \in \Gamma(N)$. In summary, we have shown that $\left(A_{Z}, H_{Z}, \kappa(\gamma, Z)\right.$, $\left.\left\{F_{Z}\left(e_{i} / N\right), F_{Z}\left(f_{j} / N\right)\right\}\right)$ is a real principally polarized Abelian variety with (compatible) level $N$ structure, and that the isomorphism $\phi$ preserves both the real structure and the level structure.

Finally, an isomorphism $\phi: \mathcal{A} \rightarrow \mathcal{A}^{\prime}$ (6.2.1) between real principally polarized Abelian varieties with level $N=4 m$ structures determines a cohomological equivalence between the corresponding 1-cocycles as follows. Choose $Z, Z^{\prime} \in \mathfrak{h}_{n}$ and $\gamma, \gamma^{\prime} \in \Gamma(4 m)$ for $\mathcal{A}$ and $\mathcal{A}^{\prime}$ respectively, as above, and set

$$
g={ }^{t}\left(F_{Z}^{-1} \circ \phi \circ F_{Z^{\prime}}\right) .
$$

Then $g \in \Gamma(4 m)$ by Section 6.2(d). Since $\phi \kappa \phi^{-1}=\kappa^{\prime}$, diagrams 6.3.1 and 6.4.2 give

$$
{ }^{t} g^{t} \gamma^{t} I_{-}{ }^{t} g^{-1}={ }^{t} \gamma^{\prime} I_{-}
$$

or $\gamma^{\prime}=\tilde{g}^{-1} \gamma g$. Hence $\left[f_{\gamma}\right]=\left[f_{\gamma^{\prime}}\right]$ in $H^{1}(\langle\tau\rangle, \Gamma(4 m))$.

\subsection{PROOF OF THEOREM 6.6.}

Let $\mathcal{I}$ denote the moduli space of isomorphism classes of real Abelian varieties with principal polarization and level $N$ structure. Let $S=\mathfrak{h}_{n}^{\tau \Gamma(N)}$ denote the set of $\Gamma(N)$-real points in $\mathfrak{h}_{n}$ and let $\Gamma(N) \backslash S$ denote its image in $X=\Gamma(N) \backslash \mathfrak{h}_{n}$. Let $\Phi: S \rightarrow \mathcal{I}$ be the association

$$
Z \mapsto\left(A_{Z}, H_{Z}, \kappa_{Z},\left\{F_{Z}\left(e_{i} / N\right), F_{Z}\left(f_{j} / N\right)\right\}\right) .
$$

Here, $\kappa_{Z}: \mathbb{C}^{n} \rightarrow \mathbb{C}^{n}$ is the real structure given by (6.4.2), that is, $\kappa(M)={ }^{t}(C Z+D) \bar{M}$ where $\gamma=\left(\begin{array}{ll}A & B \\ C & D\end{array}\right) \in \Gamma(N)$ is the unique element such that $\gamma \cdot Z=-\bar{Z}$. Then Proposition 7.5 says that $\Phi$ is surjective. If $Z, Z^{\prime} \in S$ and if $Z^{\prime}=\gamma \cdot Z$ for some $\gamma \in \Gamma(N)$ then by (6.3.1) the real Abelian variety $\left(A_{Z}, H_{Z}, \kappa_{Z},\left\{F_{Z}\left(e_{i} / N\right), F_{Z}\left(f_{i} / N\right)\right\}\right)$ is isomorphic to $\left(A_{Z^{\prime}}, H_{Z^{\prime}}, \kappa_{Z^{\prime}},\left\{F_{Z^{\prime}}\left(e_{i} / N\right), F_{Z^{\prime}}\left(f_{i} / N\right)\right\}\right)$. Therefore $\Phi$ passes to a mapping $\Gamma(N) \backslash S \rightarrow \mathcal{I}$.

On the other hand, if $Z, \Omega \in S$ and if there is an isomorphism $\psi: \mathcal{A}_{\Omega} \rightarrow \mathcal{A}_{Z}$ then by (6.3.1) there exists $h \in \mathrm{Sp}(2 n, Z)$ such that $\Omega=h \cdot Z$. Since the mapping $\psi$ also preserves the level structures, it follows also from (6.3.1) that $h \in \Gamma(N)$. Hence the mapping $\Gamma(N) \backslash S \rightarrow \mathcal{I}$ is also injective. By Corollary 4.4, the quotient $\Gamma(N) \backslash S$ is precisely the variety $X_{\mathrm{R}}$ of real points in $X$.

\section{Baily-Borel Compactification}

Let $\overline{\mathfrak{h}}_{n}$ denote the Satake partial compactification of $\mathfrak{h}_{n}$ which is obtained by attaching all rational boundary components, with the Satake topology. The group 
$\operatorname{Sp}(2 n, Q)$ acts on $\overline{\mathfrak{h}}_{n}$, the involution $\tau: \mathfrak{h}_{n} \rightarrow \mathfrak{h}_{n}$ extends to $\overline{\mathfrak{h}}_{n}$, and $\tau(g x)=\tau(g) \tau(x)$ for any $g \in \operatorname{Sp}(2 n, \mathbb{Q})$ and every $x \in \overline{\mathfrak{h}}_{n}$. Denote by $\pi: \overline{\mathfrak{h}}_{n} \rightarrow \Gamma(4 m) \backslash \overline{\mathfrak{h}}_{n}=V$ the projection to the Baily-Borel compactification of $X$. The involution $\tau$ passes to complex conjugation $\tau: V \rightarrow V$, whose fixed points we denote by $V_{\mathbb{R}}$. Clearly $X_{\mathbb{R}} \subset V_{\mathbb{R}}$. Define $\bar{X}_{\mathbb{R}}$ to be the closure of $X_{\mathbb{R}}$ in $V_{\mathbb{R}}$.

In [Sh2], Shimura shows that the $\Gamma(N)$-automorphic forms on $\mathfrak{h}_{n}$ are generated by those automorphic forms with rational Fourier coefficients. It follows that:

THEOREM 8.1. There exists a natural rational structure on the Baily-Borel compactification $V$ of $X$ which is compatible with the real structure defined by $\tau$.

The Baily-Borel compactification $V$ is stratified by finitely many strata of the form $\pi(F)$ where $F \subset \overline{\mathfrak{h}}_{n}$ is a rational boundary component. Each such $F$ is isomorphic to some Siegel space $\mathfrak{h}_{k}$, in which case we say the stratum $\pi(F)$ is a boundary stratum of rank $k$. Let $V^{r}$ denote the union of all boundary strata of rank $n-r$. In Proposition 9.2 we will prove that

$$
V_{\mathrm{R}}^{0} \cup V_{\mathrm{R}}^{1} \subset \bar{X}_{\mathrm{R}} \subset V_{\mathrm{R}}
$$

where $V_{\mathbb{R}}^{r}=V^{r} \cap V_{\mathbb{R}}$.

As in Section 3.3, if $F$ is a rational boundary component of $\mathfrak{h}_{n}$ which is preserved by the involution $\tau$, and if $\gamma \in \Gamma(4 m)$, we define the set of $\gamma$-real points of $F$ to be

$$
F^{\tau \gamma}=\{x \in F \mid \tau(x)=\gamma x\} .
$$

Then $\pi\left(F^{\tau \gamma}\right) \subset V_{\mathbb{R}}$. A $\Gamma(4 m)$-real boundary pair $(F, \gamma)$ (of rank $q$ ) consists of a rational boundary component $F$ (of rank $q$ ) and an element $\gamma \in \Gamma(4 m)$ such that $F^{\tau \gamma} \neq \phi$. (Hence $\tilde{F}=\gamma F$.) We say two real boundary pairs $(F, \gamma)$ and $\left(F_{1}, \gamma_{1}\right)$ are equivalent if the resulting locus of real points $\pi\left(F^{\tau \gamma}\right)=\pi\left(F_{1}^{\tau \gamma_{1}}\right)$ coincide. If $(F, \gamma)$ is a real boundary pair and if $g \in \Gamma(4 m)$ then $\left(g F, \tilde{g} \gamma g^{-1}\right)$ is an equivalent real boundary pair.

\subsection{STANDARD BOUNDARY COMPONENTS}

Fix an integer $q$ with $1 \leqslant q<n$. The Siegel upper halfspace $\mathfrak{h}_{q}$ is attached to $\mathfrak{h}_{n}$ as a limit of matrices in $M_{n \times n}(\mathrm{C})$ by

$$
Z \mapsto \lim _{Y \rightarrow \infty}\left(\begin{array}{cc}
Z & 0 \\
0 & i Y
\end{array}\right)
$$

See (3.2.2). Here, $Y \in C_{n-q}$ is a positive definite symmetric matrix of order $n-q$, and the limit is taken as $Y \rightarrow \infty$, meaning that all the eigenvalues of $Y$ converge to $\infty$. Denote this mapping by $\phi: \mathfrak{h}_{q} \rightarrow \overline{\mathfrak{h}}_{n}$. Its image $F_{q}=\phi\left(\mathfrak{h}_{q}\right) \subset \overline{\mathfrak{h}}_{n}$ is called the standard 
boundary component of (maximal) rank $q$. The normalizer in $\operatorname{Sp}(2 n, Z)$ of $F_{q}$ is the parabolic subgroup

$$
P_{q}=\left(\begin{array}{cc|cc}
A & 0 & B & * \\
* & * & * & * \\
\hline C & 0 & D & * \\
0 & 0 & 0 & *
\end{array}\right)
$$

with unipotent radical

$$
\mathcal{U}\left(P_{q}\right)=\left(\begin{array}{cc|cc}
I_{q} & 0 & 0 & b \\
0 & I_{n-q} & { }^{t} b & d \\
\hline 0 & 0 & I_{q} & -{ }^{t} a \\
0 & 0 & 0 & I_{n-q}
\end{array}\right)
$$

and Levi factor $L\left(P_{q}\right)=G_{h} G_{\ell}$ with

$$
G_{h}=\left(\begin{array}{cc|cc}
A & 0 & B & 0 \\
0 & I_{n-q} & 0 & 0 \\
\hline C & 0 & D & 0 \\
0 & 0 & 0 & I_{n-q}
\end{array}\right) \text { and } G_{\ell}=\left(\begin{array}{cc|cc}
I_{q} & 0 & 0 & 0 \\
0 & T & 0 & 0 \\
\hline 0 & 0 & I_{q} & 0 \\
0 & 0 & 0 & { }^{t} T^{-1}
\end{array}\right)
$$

being the 'Hermitian' and 'linear' factors respectively, where $\left(\begin{array}{cc}A & B \\ C & D\end{array}\right) \in \mathrm{Sp}(2 q, \mathbb{R})$ and $T \in \mathrm{GL}_{n-q}(\mathrm{R})$. The subgroup $\mathcal{U}\left(P_{q}\right) G_{\ell}$ is normal in $P_{q}$ and we denote by

$$
v: P_{q} \rightarrow G_{h} \cong \operatorname{Sp}(2 q, \mathbb{R})
$$

the projection to the quotient. Then $v$ commutes with the involution $\tau$.

The boundary component $F_{q}$ is preserved by $\tau$. The set

$$
F_{q}^{\tau}=\phi\left(i C_{q}\right)=\{\phi(i Y) \mid Y>0\}
$$

of $I$-real points in $F_{q}$ is just the set of $\tau$-fixed points in $F_{q}$ and it may be canonically identified with the cone of positive definite matrices of order $q$. Denote by $i I_{q}$ its canonical basepoint. The boundary component $F_{q}$ is attached to $\mathfrak{h}_{n}$ so that this cone $\phi\left(i C_{q}\right)$ is contained in the closure of the cone $i C_{n}$.

PROPOSITION 8.3. Let $(F, \gamma)$ be a $\Gamma(4 m)$-real boundary pair of rank $q$. Then there exists $a \in \operatorname{Sp}(2 n, Z)$ so that $a\left(F_{q}\right)=F$ and

$$
\tilde{a}^{-1} \gamma a=\left(\begin{array}{cc}
A & B \\
0 & { }^{t} A^{-1}
\end{array}\right) \in \operatorname{ker}(v)
$$


Moreover, we may take $B=0$, that is, there exists $\gamma^{\prime} \in \Gamma(4 m)$ and $g \in \operatorname{Sp}(2 n, Z)$ so that $F^{\tau \gamma^{\prime}}=F^{\tau \gamma}, g\left(F_{q}\right)=F$, and so that

$$
\tilde{g}^{-1} \gamma^{\prime} g=\left(\begin{array}{cc}
A & 0 \\
0 & A^{-1}
\end{array}\right) \in \operatorname{ker}(v)
$$

Proof 8.4. Choose $b \in \operatorname{Sp}(2 n, Z)$ so that $b F_{q}=F$. Then

$$
w=\tilde{b}^{-1} \gamma b=\tilde{b}^{-1} b \cdot b^{-1} \gamma b \in \Gamma(2) .
$$

Since it also preserves the standard boundary component $F_{q}$, we have: $w \in P_{q}$.

The Hermitian part $v(w) \in \mathrm{Sp}(2 n, Z)$ acts on the standard boundary component $F_{q} \cong \mathfrak{h}_{q}$. By Lemma $4.8, v(\tilde{w}) v(w) \in \Gamma(4)$ which is torsion-free. By assumption, the set of $v(w)$-real points $F_{q}^{\tau v(w)}$ is nonempty. Choose a point $x \in F_{q}^{\tau v(w)}$ whose stabilizer in $\operatorname{Sp}(2 q, Z)$ consists of $\pm I$ : by Lemma 3.4 such points exist and are even dense in $F_{q}^{\tau v(w)}$. Then, by Proposition 4.10 there exists $h \in \operatorname{Sp}(2 q, Z)$ such that $\tilde{h} h^{-1}=v(w)$ and $F_{q}^{\tau v(w)}=h\left(i C_{q}\right)$. Let us identify the element $h$ with its image in $\operatorname{Sp}(2 n, Z)$ as in (8.2.1) and set $v=\tilde{h}^{-1} w h$. Then $v(v)=1$. The following diagram may help to sort out these various transformations.

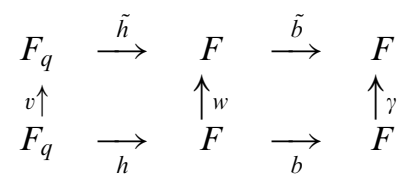

Then the element $a=b h \in \mathrm{Sp}(2 n, \mathrm{Z})$ has the desired properties, that is, $a \cdot F_{q}=F$ and $v(v)=1$ where $v=\tilde{a}^{-1} \gamma a \in \Gamma(2)$.

Now let us prove the 'moreover' part of Proposition 8.3. By Lemma 4.8, both $\tilde{\gamma} \gamma^{-1}$ and $\tilde{\gamma} \gamma$ are in $\Gamma(8 m)$. Then $\tilde{\gamma} \gamma=\tilde{a} \tilde{v} v \tilde{a}^{-1} \in \Gamma(8 m)$, hence $\tilde{v} v \in \Gamma(8 m)$. Calculating $\tilde{v} v \equiv I(\bmod 8 m)$ gives

$$
A B \equiv B^{t} A^{-1}(\bmod 8 m) \text { and } A^{2} \equiv I(\bmod 8 m) .
$$

Since $v=\tilde{a}^{-1} a a^{-1} \gamma a \in \Gamma(2)$ the matrix $A$ is integral and $B$ is even, so

$$
x=\left(\begin{array}{cc}
I & -\frac{1}{2} A^{-1} B \\
0 & I
\end{array}\right)
$$

is integral. Since $v \in \operatorname{ker}(v)$ we see that $A=\left(\begin{array}{c}* 0 \\ * *\end{array}\right)$ and $B=\left(\begin{array}{l}0 * \\ * *\end{array}\right)$ from which it follows that $x \in \mathcal{U}\left(P_{q}\right) \subset \operatorname{ker}(v)$. Set $g=a x$ and set $u^{\prime}=\tilde{x}^{-1} v x$. Direct computation with the matrices for $x$ and $v$ gives

$$
u^{\prime}=\left(\begin{array}{cc}
A & B^{\prime} \\
0 & A^{-1}
\end{array}\right)
$$


where $B^{\prime}=\frac{1}{2} B-\frac{1}{2} A^{-1} B^{t} A^{-1}$. Using (8.4.1) gives $B^{\prime} \equiv 0(\bmod 4 m)$. The following diagram may help to explain these transformations.

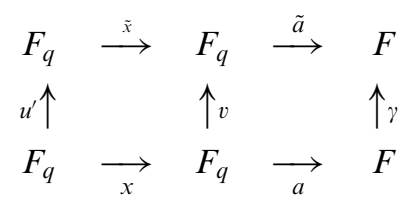

Now decompose $u^{\prime}=u u_{2}$ where

$$
u=\left(\begin{array}{cc}
A & 0 \\
0 & { }^{t} A^{-1}
\end{array}\right) \text { and } u_{2}=\left(\begin{array}{cc}
I & A^{-1} B^{\prime} \\
0 & I
\end{array}\right) \in \operatorname{ker}(v)
$$

Set $\gamma^{\prime}=\tilde{g} u g^{-1}$. We will verify that $g$ and $\gamma^{\prime}$ satisfy the conclusions of Lemma 8.3.

First note that $u_{2} \in \Gamma(4 m)$ since $B^{\prime} \equiv 0(\bmod 4 m)$. Then $\gamma^{\prime} \in \Gamma(4 m)$ because

$$
\gamma=\tilde{a} v a^{-1}=\tilde{g} u^{\prime} g^{-1}=\left(\tilde{g} u g^{-1}\right)\left(g u_{2} g^{-1}\right)=\gamma^{\prime}\left(g u_{2} g^{-1}\right) \in \Gamma(4 m) .
$$

We have already verified that $g F_{q}=F$ and that $u=\tilde{g}^{-1} \gamma^{\prime} g$ has the desired form. Since $u_{2}$ acts trivially on $F_{q}$ we see that $y \in F^{\prime}$ iff

$$
\tilde{y}=\gamma^{\prime} y=\tilde{g} u g^{-1} y=\tilde{g} u u_{2} g^{-1} y=\gamma y
$$

iff $y \in F^{\tau \gamma}$. Hence $F^{\tau \gamma^{\prime}}=F^{\tau \gamma}$.

\subsection{SOME NEARBY BOUNDARY COMPONENTS}

Fix $r$ with $q \leqslant r \leqslant n$ and set $s=n-r$. Define

$$
j_{r}=\left(\begin{array}{cc|cc}
I_{r} & 0 & 0 & 0 \\
0 & 0 & 0 & I_{s} \\
\hline 0 & 0 & I_{r} & 0 \\
0 & -I_{s} & 0 & 0
\end{array}\right) \text { so } j_{r}^{2}=\left(\begin{array}{cc|cc}
I_{r} & 0 & 0 & 0 \\
0 & -I_{s} & 0 & 0 \\
\hline 0 & 0 & I_{r} & 0 \\
0 & 0 & 0 & -I_{s}
\end{array}\right) \text {. }
$$

Then $\tilde{j}_{r}=j_{r}^{-1}$. Although $j_{r} \notin P_{q}$, its square $j_{r}^{2}$ preserves $F_{q}$ and in fact it acts as the identity on $F_{q}$. Let $E_{q, r}=j_{r}\left(F_{q}\right)=j_{r}^{-1}\left(F_{q}\right)$ and let $Q_{q, r}$ be its normalizing maximal parabolic subgroup: it is $j_{r}$-conjugate to $P_{q}$. (If $r=n$ then $j_{r}$ is the identity and $E_{q, r}=F_{q}$ ). The involution $\tau$ preserves the boundary component $E_{q, r}$ and the set of $\tau$-fixed points

$$
E_{q, r}^{\tau}=\left\{x \in E_{q, r} \mid \tilde{x}=x\right\}=j_{r} \cdot F_{q}^{\tau}
$$

is contained in the closure of $\mathfrak{h}_{n}^{\tau}=i C_{n}$ as follows. Although $j_{r}$ does not preserve the cone $i C_{n}$, it does preserve the sub-cone $S_{q, r} \subset i C_{n}$ of elements

$$
\left(\begin{array}{ccc}
i Y_{1} & 0 & 0 \\
0 & i Y_{2} & 0 \\
0 & 0 & i Y_{3}
\end{array}\right) \in \mathfrak{h}_{n},
$$


where $Y_{1} \in C_{q}, Y_{2} \in C_{r-q}$, and $Y_{3} \in C_{s}$. Moreover, $F_{q}^{\tau}$ is contained in the closure of $S_{q, r}$. Therefore

$$
E_{q, r}^{\tau}=j_{r} F_{q}^{\tau} \subset j_{r} \overline{S_{q, r}}=\overline{S_{q, r}} \subset \overline{i C_{n}} .
$$

PROPOSITION 8.6. Let $(F, \gamma)$ be a $\Gamma(4 m)$-real boundary pair of rank q. Let $g \in \operatorname{Sp}(2 n, Z)$. Suppose that $g\left(F_{q}\right)=F$ and that $u=\tilde{g}^{-1} \gamma g \in \operatorname{ker}(v)$. Suppose also that there exists $r$ with $q \leqslant r \leqslant n$ so that

$$
\tilde{j}_{r}^{-1} u j_{r}=j_{r} u j_{r} \in \Gamma(4 m) .
$$

Define $\omega=\tau\left(g j_{r}\right)\left(g j_{r}\right)^{-1}$. Then $\omega \in \Gamma(4 m)$ and

$$
F^{\tau \gamma}=F^{\tau \omega} \subset \overline{\mathfrak{h}_{\mathfrak{n}}^{\tau \omega}} .
$$

Consequently the resulting set $\pi\left(F^{\tau \gamma}\right)$ of real points is contained in the closure $\bar{X}_{\mathbb{R}}$.

Proof 8.7. Calculate

$$
\omega=\tau\left(g j_{r}\right)\left(g j_{r}\right)^{-1}=\gamma\left(\left(g j_{r}\right)\left(j_{r} u j_{r}\right)^{-1}\left(g j_{r}\right)^{-1}\right) \in \Gamma(4 m)
$$

(using the fact that $\tilde{j}_{r}=j_{r}^{-1}$ ) which proves the first statement. Since $u \in P_{q}$ acts trivially on $F_{q}$, the same is true of $j_{r}^{2} u$. Hence, $x \in F^{\tau \omega}$ if and only if

$$
\tilde{x}=\omega x=\tau\left(g j_{r}\right) j_{r}^{-1}\left(g^{-1} x\right)=\tau\left(g j_{r}\right) j_{r}^{-1} j_{r}^{2} u\left(g^{-1} x\right)=\tau\left(g j_{r}\right) \tau\left(g j_{r}\right)^{-1} \gamma x=\gamma x
$$

which holds if and only if $x \in F^{\tau \gamma}$. The following diagram may help in placing these elements,

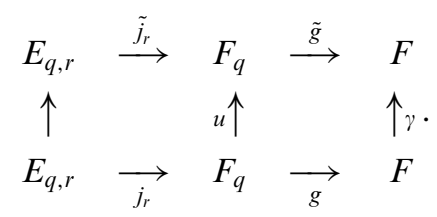

We claim that $F^{\tau \omega}=g j_{r}\left(E_{q, r}^{\tau}\right)$. In fact, $x \in E_{q, r}^{\tau}$ if and only if $\tilde{x}=x$ which holds iff

$$
\tau\left(g j_{r}\right) \tilde{x}=\tau\left(g j_{r}\right) x=\tau\left(g j_{r}\right)\left(g j_{r}\right)^{-1}\left(g j_{r}\right) x=\omega\left(g j_{r}\right) x
$$

which holds iff $\left(g j_{r}\right) x \in F^{\tau \omega}$. Similarly, $g j_{r}\left(\mathfrak{h}_{n}^{\tau}\right)=\mathfrak{h}_{n}^{\tau \omega}$. Hence

$$
F^{\tau \omega}=g j_{r}\left(E_{q, r}^{\tau}\right) \subset g j_{r} \overline{\mathfrak{h}_{n}^{\tau}}=\overline{\mathfrak{h}_{n}^{\tau \omega}}
$$

using (8.5.1).

\section{Corank One Strata}

9.1. In this section we specialize to the case $q=n-1$, that is, we consider only the boundary strata of maximal rank. As in Section $8, F_{n-1}$ denotes the standard 
boundary component of corank $1, P_{n-1}$ denotes its normalizing parabolic subgroup and $G_{h}$ and $G_{\ell}$ refer to the Hermitian and linear factors (8.2.1) of the Levi quotient $L\left(P_{n-1}\right)$.

THEOREM 9.2. Let $F$ be a proper rational boundary component of $\mathfrak{h}_{n}$ with (maximal) rank $n-1$, let $\gamma \in \Gamma(4 m)$, and suppose that $F^{\tau \gamma} \neq \phi$. Then $F^{\tau \gamma}$ is contained in the closure of the set $\mathfrak{h}_{n}^{\tau \Gamma(4 m)}$ of $\Gamma(4 m)$-real points of $\mathfrak{h}_{n}$.

Proof 9.3. By Proposition 8.3, there exists $\gamma^{\prime} \in \Gamma(4 m)$ and $g \in \operatorname{Sp}(2 n, Z)$ so that $g F_{n-1}=F, F^{\tau \gamma}=F^{\tau \gamma^{\prime}}$ and so that

$$
u=\tilde{g}^{-1} \gamma^{\prime} g=\left(\begin{array}{cc}
A & 0 \\
0 & { }^{t} A^{-1}
\end{array}\right) \in \operatorname{ker}(v) .
$$

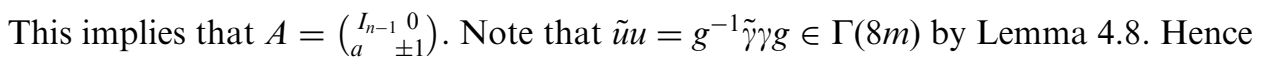
$a \equiv 0(\bmod 4 m)$.

If the plus sign occurs then this says that $u \in \Gamma(4 m)$. Let $\omega=\tilde{g} g^{-1}$. Then Proposition 8.6 (with $q=n-1$ and $r=n$ ) implies that $F^{\tau \gamma^{\prime}} \subset \overline{\mathfrak{h}_{n}^{\tau \omega}}$.

If the minus sign occurs then

$$
j_{n-1} u j_{n-1}=\left(\begin{array}{cc|cc}
I & 0 & 0 & 0 \\
0 & 1 & 0 & 0 \\
\hline 0 & -{ }^{t} a & I & 0 \\
-a & 0 & 0 & 1
\end{array}\right) \in \Gamma(4 m)
$$

so we may apply Proposition 8.6 (with $q=r=n-1$ ) to conclude that $F^{\tau \gamma^{\prime}} \subset \overline{\mathfrak{h}_{n}^{\tau \omega}}$ where $\omega=\tau\left(g j_{n-1}\right)\left(g j_{n-1}\right)^{-1}$.

\section{The Principal Congruence Group $\Gamma\left(2^{k}\right)$}

10.1. Throughout this section we let $\Gamma=\Gamma\left(2^{k}\right) \subset \operatorname{Sp}(2 n, Z)$ be the principal congruence subgroup of level $2^{k}$ with $k \geqslant 2$. As in Section 8, let $X=X_{\mathrm{C}}=\Gamma \backslash \mathfrak{h}_{n}$ and let $V=V_{\mathrm{C}}=\Gamma \backslash \overline{\mathfrak{G}}_{n}$ be its Baily-Borel compactification with projection $\pi: \overline{\mathfrak{h}}_{n} \rightarrow V$. Let $\bar{X}_{\mathbb{R}}$ be the closure of $X_{\mathbb{R}}$ in $V$. In this section we will prove that $\bar{X}_{\mathbb{R}}=V_{\mathbb{R}}$. The proof of the following lemma will appear in Section 10.7.

LEMMA 10.2. Let $A \in \mathrm{GL}(n, Z)$. Suppose that

$$
A \equiv I(\bmod 2) \text { and } A^{2} \equiv I\left(\bmod 2^{k+1}\right) .
$$

Then there exists $p \in \mathrm{GL}(n, Z)$ so that

$$
p^{-1} A p \equiv\left(\begin{array}{llll} 
\pm 1 & & & \\
& \pm 1 & & \\
& & \ldots & \\
& & & \pm 1
\end{array}\right)\left(\bmod 2^{k}\right) .
$$


Moreover if the matrix of $A$ with respect to the standard basis of $Z^{n}$ is $\left(\begin{array}{c}I_{q} \\ * *\end{array}\right)$ then it is possible to choose $p$ to be of the form $p=\left(\begin{array}{l}I_{q} \\ * *\end{array}\right)$.

LEMMA 10.3. Let $(F, \gamma)$ be a real boundary pair of rank $q$. Then there exists $r \geqslant q$, there exists $\gamma^{\prime} \in \Gamma\left(2^{k}\right)$ and there exists $g \in \operatorname{Sp}(2 n, Z)$ such that $F^{\tau \gamma}=F^{\tau \gamma^{\prime}}, g F_{q}=F$, $\tilde{g}^{-1} \gamma^{\prime} g \in \operatorname{ker}(v)$, and so that

$$
\tilde{g}^{-1} \gamma^{\prime} g \equiv\left(\begin{array}{cc|cc}
I_{r} & 0 & 0 & 0 \\
0 & -I_{s} & 0 & 0 \\
\hline 0 & 0 & I_{r} & 0 \\
0 & 0 & 0 & -I_{s}
\end{array}\right) \quad\left(\bmod 2^{k}\right)
$$

where $s=n-r$.

Proof 10.4. By Proposition 8.3 there exists $\gamma^{\prime} \in \Gamma\left(2^{k}\right)$ and there exists $a \in \mathrm{Sp}(2 n, Z)$ so that $F^{\tau \gamma}=F^{\tau \gamma^{\prime}}, a F_{q}=F$, and

$$
u=\tilde{a}^{-1} \gamma^{\prime} a=\left(\begin{array}{cc}
A & 0 \\
0 & { }^{t} A^{-1}
\end{array}\right) \in \operatorname{ker}(v) .
$$

Then $\tilde{\gamma}^{\prime} \gamma^{\prime}=a u^{2} a^{-1} \in \Gamma\left(2^{k+1}\right)$ by Lemma 4.8 , hence $A^{2} \equiv I\left(\bmod 2^{k+1}\right)$. Moreover, $A \in \Gamma(2)$ and $A=\left(\begin{array}{l}I_{q} 0 \\ * * *\end{array}\right)$. Let $p \in \operatorname{GL}(n, Z)$ be the change of basis provided by Lemma 10.2. Then $p^{-1} A p=\left(\begin{array}{l}I_{q} \\ * \\ * *\end{array}\right)$ and (after re-ordering the coordinates if necessary), $p^{-1} A p \equiv\left(\begin{array}{cc}I_{q} & 0 \\ 0 & -I_{s}\end{array}\right)\left(\bmod 2^{k}\right)$ for some $r \geqslant q$. Set $h=\left(\begin{array}{cc}p & 0 \\ 0 & p^{-1}\end{array}\right) \in \operatorname{ker}(v)$. Set $g=a h$.

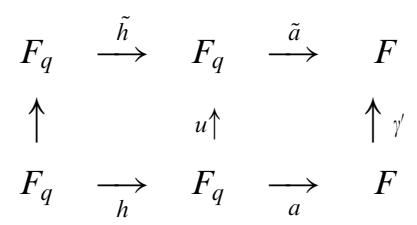

Then $\tilde{h}^{-1} u h=\tilde{g}^{-1} \gamma^{\prime} g \in \operatorname{ker}(v)$ and $\tilde{g}^{-1} \gamma^{\prime} g$ has the desired form (10.3.1).

THEOREM 10.5. Let $(F, \gamma)$ be a real boundary pair. Then there exists $\gamma_{1} \in \Gamma\left(2^{k}\right)$ so that the set $F^{\tau \gamma}=F^{\tau \gamma_{1}}$ of $\gamma$-real points is contained in the closure $\overline{\mathfrak{h}_{n}^{\tau \gamma_{1}}}$.

Proof 10.6. Set $q=\operatorname{rank}(F)$. By Lemma 10.3, there exists $\gamma_{1} \in \Gamma\left(2^{k}\right)$ and there exists $g \in \operatorname{Sp}(2 n, Z)$ so that $F^{\tau \gamma}=F^{\tau \gamma_{1}}$, so that $g\left(F_{n-1}\right)=F$ and so that $u=\tilde{g}^{-1} \gamma_{1} g$ lies in $\operatorname{ker}(v)$ and has the form (10.3.1), for some $r \geqslant q$. Therefore $j_{r} u j_{r} \equiv I\left(\bmod 2^{k}\right)$ so Proposition 8.6 may be applied.

\subsection{PROOF OF LEMMA 10.2.}

The lemma is equivalent to the following statement. Suppose $M$ is a free $Z$-module of rank $n$. Let $\alpha: M \rightarrow M$ be an automorphism such that $(\alpha-I) M \subset 2 M$ and $\left(\alpha^{2}-I\right) M \subset 2^{k+1} M$, that is, $\alpha \equiv I(\bmod 2)$ and $\alpha^{2} \equiv I\left(\bmod 2^{k+1}\right)$. Then there exists a basis $x_{1}, x_{2}, \ldots, x_{n}$ of $M$ so that $\alpha\left(x_{i}\right)= \pm x_{i} \in 2^{k} M$ for $i=1,2, \ldots, n$. 
This statement will be proven by induction on the rank of $M$. The case of rank 1 is obvious, so suppose that $M$ has rank $n$. We will show that there exists a basis $\left\{x_{1}, x_{2}, \ldots, x_{n}\right\}$ of $M$ so that $\alpha\left(x_{1}\right) \equiv \pm x_{1}\left(\bmod 2^{k}\right)$. If $\operatorname{ker}(\alpha-I)$ is not trivial or if $\operatorname{ker}(\alpha+I)$ is not trivial then any primitive $x$ within this kernel may be extended to a basis. Therefore we may assume that both $(\alpha-I) M$ and $(\alpha+I) M$ have maximal rank.

By elementary divisor theory there exists a basis $x_{1}, x_{2}, \ldots, x_{n}$ of $M$ and integers $d_{1}, d_{2}, \ldots, d_{n}$ so that $d_{1}\left|d_{2}\right| \ldots \mid d_{n}$ and so that $d_{1} x_{1}, d_{2} x_{2}, \ldots, d_{n} x_{n}$ is a basis of $(\alpha-I) M$. Similarly there exists another basis $y_{1}, y_{2}, \ldots, y_{n}$ of $M$ and integers $e_{1}\left|e_{2}\right| \ldots \mid e_{n}$ so that $e_{1} y_{1}, e_{2} y_{2}, \ldots, e_{n} y_{n}$ is a basis for the submodule $(\alpha+I) M \subset M$.

We claim that either $\alpha\left(x_{1}\right) \equiv-x_{1}\left(\bmod 2^{k}\right)$ or $\alpha\left(y_{1}\right) \equiv y_{1}\left(\bmod 2^{k}\right)$. First note that $(\alpha+I) d_{1} x_{1} \equiv 0\left(\bmod 2^{k+1}\right)$ since $d_{1} x_{1} \in(\alpha-I) M$. If $d_{1}$ is odd this implies $\alpha x_{1}+x_{1} \equiv 0\left(\bmod 2^{k+1}\right)$. If $d_{1} / 2$ is odd, it implies that $\alpha x_{1}+x_{1} \equiv 0\left(\bmod 2^{k}\right)$. Similarly, if $e_{1}$ is odd or if $e_{1} / 2$ is odd then $\alpha y_{1}-y_{1} \equiv 0\left(\bmod 2^{k}\right)$. However, $(\alpha-I) M+(\alpha+I) M=2 M$ so the highest power of 2 which divides $\operatorname{gcd}\left(d_{1}, e_{1}\right)$ is $2^{1}$. Therefore one of these four cases must occur, which proves the claim.

By switching the $x$ 's with the $y$ 's if necessary, we arrive at a basis $x_{1}, x_{2}, \ldots, x_{n}$ of $M$ such that $\alpha\left(x_{1}\right) \equiv \pm x_{1}\left(\bmod 2^{k}\right)$. Write $M=M_{1} \oplus M_{2}$ where $M_{1}=Z x_{1}$ and $M_{2}=\sum_{i \geqslant 2} Z x_{i}$. With respect to this decomposition, $\alpha$ has the matrix $\left(\begin{array}{l}\alpha_{11} \alpha_{12} \\ \alpha_{21} \alpha_{22}\end{array}\right)$ where $\alpha_{11} \equiv \pm 1\left(\bmod 2^{k}\right)$, and where $\alpha_{22}: M_{2} \rightarrow M_{2}$. We claim that $\alpha_{22} \equiv I(\bmod 2)$ and $\alpha_{22}^{2} \equiv I\left(\bmod 2^{k+1}\right)$. Since $\alpha \equiv I(\bmod 2)$ we have

$$
\alpha\left(\begin{array}{c}
0 \\
m_{2}
\end{array}\right)=\left(\begin{array}{c}
\alpha_{12} m_{2} \\
\alpha_{22} m_{2}
\end{array}\right) \equiv\left(\begin{array}{c}
0 \\
m_{2}
\end{array}\right)(\bmod 2)
$$

hence $\alpha_{12} \equiv 0(\bmod 2)$ and $\alpha_{22} \equiv I(\bmod 2)$. Also,

$$
\alpha\left(\begin{array}{c}
x_{1} \\
0
\end{array}\right)=\left(\begin{array}{c}
\alpha_{11} x_{1} \\
\alpha_{21} x_{1}
\end{array}\right) \equiv\left(\begin{array}{c} 
\pm x_{1} \\
0
\end{array}\right)\left(\bmod 2^{k}\right)
$$

hence $\alpha_{21} \equiv 0\left(\bmod 2^{k}\right)$. Similarly,

$$
\alpha^{2}\left(\begin{array}{c}
0 \\
m_{2}
\end{array}\right)=\left(\begin{array}{c}
* \\
\alpha_{21} \alpha_{12} m_{2}+\alpha_{22}^{2} m_{2}
\end{array}\right) \equiv\left(\begin{array}{c}
0 \\
m_{2}
\end{array}\right)\left(\bmod 2^{k+1}\right) .
$$

But $\alpha_{12} \equiv 0(\bmod 2)$ and $\alpha_{21} \equiv 0\left(\bmod 2^{k}\right)$ so the first term in this sum is congruent to $0\left(\bmod 2^{k+1}\right)$, hence $\alpha_{22}^{2} m_{2} \equiv m_{2}\left(\bmod 2^{k+1}\right)$ as claimed.

Therefore we may apply induction to the pair $\left(M_{2}, \alpha_{22}\right)$ to obtain a basis, which we again denote by $x_{2}, x_{3}, \ldots, x_{n}$ such that $\alpha_{22}\left(x_{j}\right) \equiv \pm x_{j}\left(\bmod 2^{k}\right)$ for $j \geqslant 2$. Hence, for $j \geqslant 2$, there are integers $a_{j}$ and a sign $\epsilon_{j}= \pm 1$ so that $\alpha\left(x_{j}\right) \equiv a_{j} x_{1}+\epsilon_{j} x_{j}\left(\bmod 2^{k}\right)$. Each $a_{j}$ is even since $\alpha \equiv I(\bmod 2)$. Define a new basis

$$
x_{j}^{\prime}= \begin{cases}x_{j} & \text { if } \epsilon_{j}=\epsilon_{1} \\ \epsilon_{j} x_{j}+\frac{1}{2} a_{j} x_{1} & \text { if } \epsilon_{j}=-\epsilon_{1} .\end{cases}
$$


We claim this basis has the desired property: $\alpha\left(x_{j}^{\prime}\right)= \pm x_{j}^{\prime}\left(\bmod 2^{k}\right)$. First suppose $\epsilon_{j}=+\epsilon_{1}$. Then $\alpha^{2}\left(x_{j}\right)=2 a_{j} \epsilon_{j} x_{1}+x_{j}$. Since $\alpha^{2} \equiv I\left(\bmod 2^{k+1}\right)$ we see that $a_{k} \equiv 0\left(\bmod 2^{k}\right)$. Hence $\alpha\left(x_{j}^{\prime}\right) \equiv x_{j}\left(\bmod 2^{k}\right)$ as desired. If $\epsilon_{j} \epsilon_{1}=-1$ then

$$
\begin{aligned}
\alpha\left(x_{j}^{\prime}\right) & =\epsilon_{j} \alpha\left(x_{j}\right)+\frac{1}{2} a_{j} \alpha\left(x_{1}\right) \\
& \equiv \epsilon_{j}\left(a_{j} x_{1}+\epsilon_{j} x_{j}\right)+\frac{1}{2} a_{j} \epsilon_{1} x_{1}\left(\bmod 2^{k}\right) \\
& \equiv x_{j}+\frac{1}{2} \epsilon_{j} a_{j} x_{1}\left(\bmod 2^{k}\right) \\
& =\epsilon_{j}\left(\epsilon_{j} x_{j}+\frac{1}{2} a_{j} x_{1}\right)=\epsilon_{j} x_{j}^{\prime} .
\end{aligned}
$$

This completes the construction of the desired basis.

To prove the 'moreover' part of the lemma, let $M^{\prime}=\sum_{i=1}^{q} Z e_{i}$ be the submodule of $M$ generated by the first $q$ standard basis vectors. Apply the lemma to the quotient module $\alpha: M / M^{\prime} \rightarrow M / M^{\prime}$. Choose any lift $x_{q+1}, \ldots, x_{n}$ of the resulting basis of $M / M^{\prime}$ to $M$ and define $x_{1}=e_{1}, x_{2}=e_{2}, \ldots, x_{q}=e_{q}$. With respect to this basis,

$$
\alpha\left(x_{j}\right)=\epsilon_{j} x_{j}+\sum_{j=1}^{q} a_{i j} x_{j} \text { for } i>q
$$

where $\epsilon_{i}= \pm 1$ and $a_{i j}$ is even. Set

$$
x_{i}^{\prime}= \begin{cases}x_{i} & \text { if } \epsilon_{i}=1 \\ -x_{i}+\sum_{j=1}^{q} \frac{1}{2} a_{i j} x_{j} & \text { if } \epsilon_{i}=-1\end{cases}
$$

Then $\alpha\left(x_{i}^{\prime}\right) \equiv \epsilon_{i} x_{i}^{\prime}\left(\bmod 2^{k}\right)$ and the change of basis matrix has the desired form.

\section{Acknowledgement}

The authors are grateful to the Institute for Advanced Study in Princeton, N.J. for its hospitality and support.

\section{References}

[A] Adler, A.: Antiholomorphic involutions of analytic families of Abelian varieties, Trans. Amer. Math. Soc. 254 (1979), 69-94.

[C] Comessatti, H.: Sulle varietà abeliane reali I, II, Ann. Mat. Pura. Appl. 2 (1924), 67-106 and 4 (1926), 27-72.

[GT] Goresky, M. and Tai, Y.-S.: Abelian surfaces with anti-holomorphic multiplication, Preprint, I.A.S., 2001.

[J] Jaffee, H.: Real forms of Hermitian symmetric spaces, Bull. Amer. Math. Soc. 81 (1975), 456-458.

[H] Helgason, S.: Differential Geometry, Lie Groups, and Symmetric Spaces, Academic Press, New York, 1978.

[K] Kudla, S. On the R-forms of certain algebraic varieties, Bull. Amer. Math. Soc. 81 (1975), 471-473.

[La] Lange, H. and Birkenhake, C.: Complex Abelian Varieties, Grundl. Math. Wiss. 302, Springer-Verlag, Berlin, 1992. 
[Ra1] Raševskiii, P.: The connection of the set of points of a Lie group that are fixed under one of its automorphisms, Funk. Anal. i Prilozen 6 (1972), 97-98. (English translation: Funct. Anal. Appl 6 (1972), 341-342.)

[Ra2] Raševskii, P.: A theorem on the connectedness of the subgroup of a simply connected Lie group that commutes with one of its automorphisms, Trudy Moskov. Mat. Obšc 30 (1974), 3-22. (English translation: Trans. Moscow Math. Soc. 30 (1974), 1-24.)

[Rol] Rohlfs, J.: Arithmetisch definierte Gruppen mit Galoisoperation, Invent. Math. 48 (1978), 185-205.

[Ro2] Rohlfs, J.: The Lefschetz number of an involution on the space of classes of positive definite quadratic forms, Comment. Math. Helv. 56 (1981), 272-296.

[RoS] Rohlfs, J. and Schwermer, J.: Intersection numbers of special cycles, J. Amer. Math. Soc. 6 (1993), 755-778.

[Sa] Satake, I.: Algebraic Structures of Symmetric Domains, Princeton Univ. Press, Princeton N.J., 1980.

[Si] Silhol, R.: Compactifications of moduli spaces in real algebraic geometry. Invent. Math. 107 (1992), 151-202.

[Si2] Silhol, R.: Real Abelian varieties and the theory of Comessatti, Math. Z. 181 (1982), 345-362.

[Shh] Shih, K.-Y.: Anti-holomorphic automorphisms of arithmetic automorphic function fields, Ann. of Math. 103 (1976), 81-102.

[St] Steinberg, R.: Endomorphisms of Linear Algebraic Groups, Mem. Amer. Math. Soc. 80, Amer. Math. Soc., Providence, R.I., 1968.

[Sh1] Shimura, G.: On the field of rationality for an Abelian variety, Nagoya Math. J. 45 (1972), 167-178.

[Sh2] Shimura, G.: On the Fourier coefficients of modular forms of several variables, Nachr. Akad. Wiss. Göttingen Math-Phys. 17 (1975), 261-268.

[Sh3] Shimura, G.: On the real points of an arithmetic quotient of a bounded symmetric domain, Math. Ann. 215 (1975), 135-164.

[Sh4] Shimura, G.: On abelian varieties with complex multiplication, Proc. London Math. Soc. 34 (1977), 65-86. 